$\xi^{2}=-1$

\title{
Insight into geochemistry of basaltic rocks from Mt Cameroon and characterization of the mantle source
}

\author{
Fadimatou Ngounouno Yamgouot ${ }^{1 *}$, Isaac Bertrand Gbambie Mbowou ${ }^{2}$, Ismaïla Ngounouno ${ }^{2}$, Azizi Abdoul \\ Youpoungam ${ }^{2}$, Isaac Daama ${ }^{1}$, Bernard Déruelle ${ }^{\mathrm{C}}$ \\ ${ }^{1}$ Département des Sciences de la Terre, Faculté des Sciences, Université de Ngaoundéré, B.P. 454 Ngaoundéré, Cameroun \\ ${ }_{2}^{2}$ Département des Mines et de la Géologie, Ecole de Géologie et d'Exploitation Minière (EGEM), Université de Ngaoundéré, B.P. 115 \\ Meiganga, Cameroun \\ ${ }^{3}$ Laboratoire de Magmatologie et Géochimie Inorganique et Expérimentale (MAGIE), Institut de Physique du Globe de Paris, UMR \\ 7154, Université Pierre et Marie Curie, 4, place Jussieu, 75252 Paris cédex 05, France \\ *Corresponding author E-mail: mbowou2000@yahoo.fr
}

\begin{abstract}
Alkaline volcanic activities occurred in the Mt Cameroon at the ocean-continent boundary of the Cameroon Line. It is characterized by a volcanic association of alkali basalts and hawaiites extruded during the late Miocene to Recent times. The major and trace element geochemistry of the Mt Cameroon are consistent with the fractional crystallization of olivine \pm clinopyroxene \pm plagioclase ( \pm amphibole). Petrographical and mineralogical study reveals the presence of xenocryts (olivine, clinopyroxene and spinel) in Mt Cameroon basalts. Their composition are similar to xenoliths and rocks crystals and they come from cumulates formed in the upper lithospheric mantle. Mt Cameroon magmas were generated near the boundary of garnet and spinel mantle stability domains (60-75 km depth), at the base of the lithospheric mantle that the compositions of the Mt Cameroon magmas are consistent with derivation from a infralithospheric mantle that was metasomatised by carbonatite melts. Basaltic volcanism in the Mt Cameroon occurred probably as a result of minor plume activity coupled with lithospheric extension.
\end{abstract}

Keywords: Mt Cameroon; Basalt; Xenocryst; Subcontinental; Mantle; Alkaline.

\section{Introduction}

Commonly, it is considered that all the basalts emitted on the Earth' surface, originated from the mantle after partial melting. Indeed, geochemical studies of basalts could provide essential knowledge of the mantle composition. Thus, their isotopic signature is often considered to identify the source and their trace element characteristics are used to constraint source composition as well as partial melting and fractional crystallization processes. Moreover, these data carried out an important chemical contrast between rocks emitted in oceanic domain and those emplaced in the continental domain. Despite the existence of alkaline rocks (OIB), the most common oceanic basalts are tholeiites which are silica-saturated and relatively poor in incompatible elements. In contrast, the intraplate continental basalts (alkali basalts and nephelinites) are mostly alkaline rocks which are silica-undersaturated and enriched in incompatible elements. These characteristics raise the problems of their mode of formation and the chemical composition of their sources. Moreover, during their ascent, they are susceptible to be contaminated by the lithospheric mantle and the continental crust.

The Cameroon Hot Line (CHL) is a unique within-plate volcanic province which straddles a continental margin. It consists of a chain of Cenozoic to Recent, generally alkaline volcanoes stretching from the Atlantic island of Pagalu to the interior of the African continental and oceanic area. Among the numerous hypotheses that have been proposed to explain the structure and the formation of the Cameroon Hot Line (for a comprehensive review and a discussion, see Déruelle et al. 1991), the most widely accepted structural explanation is that the CHL would be a succession of mega-tension gashes resulting from reworking during AptianAlbian times of the $N 70^{\circ} \mathrm{E}$ shear zones at the beginning of the opening of the Central Atlantic Ocean (Moreau et al. 1987).The reworking of shear zones could be linked to the occurrence of hot lines in the asthenospheric mantle (Bonatti et al. 1976).

If the dynamics and functioning of the CHL volcanic system is easily understood, the questions asked about the chemistry of the lavas, the nature of magma sources in the ocean-continent lithosphere (Bioko, Mt Cameroon, Etinde) remains still very much debated. In fact, there are many controversies about the origin of basalts located at the ocean-continent limit of the CHL. As can be seen, although basalts at the ocean-continent boundary, alkaline and chemically similar to many oceanic basalt (OIB), there remains considerable debate as to whether the source of the alkaline basalts is the subcontinental lithospheric mantle (SCLM), asthenospheric mantle, or deep mantle bound to the hot spot . In addition, whether variations in the chemical and isotopic compositions of primitive alkaline volcanic mafic rocks present at the oceancontinent boundary are the result of mixing the various final terms or the result of crustal contamination remains a difficulty to solve. Mt Cameroon is the only currently active volcano of the Cameroon Line, located at the transition area between ocean and continent. It has emitted essentially basaltic lavas (Déruelle et al. 1987), and, is associated to a small volcano (Mt Etinde) located on its SW flank, composed mainly of nephelinites (Nkoumbou et al. 1995). From a study of wehrlite and clinopyroxenite xenoliths, 
Ngounouno et al. (2005) provided evidence that portions of the lithospheric mantle beneath the Mt Cameroon are isotopically enriched. The Mt Cameroon volcano provides, therefore, an opportunity to study petrogeneis of alkali basalts in a continental and oceanic area. This work presents the petrological and geochemical data of the volcanic rocks from the Mt Cameroon in order to discuss their genesis and to constraint the mineralogy and composition of their mantle source. These data are used to develop new constraints on the origin of the Cameroon Line.

\section{Geological setting and mt cameroon volcan- ic landforms}

Mt Cameroon (Fig.1) is a Plio-quaternary volcanic massif, without any central crater, composed of lava flows and of more than 140 pyroclastic cones erected upon a horst of Paleozoic basement. Mt
Cameroon is an active volcano. It contains distinct volcanic landforms that reflect different styles of activity. Scoria cones with associated lava flows dominate the landscape; they are associated with basalt and hawaiite actvity. The present Mt Cameroon surface exposes 140 pyroclastic cones including parasitic cones, main craters and vents from which pyroclastics and lavas were ejected. They are as much as $100 \mathrm{~m}$ high, typically steep, and open on one side where lava flows were extruded. On a regional scale the outcrops of the Mt Cameroon define a subcircular-shaped region, and from the distribution of the emission points a predominantly $\mathrm{N} 40^{\circ} \mathrm{E}-\mathrm{N} 50^{\circ} \mathrm{E}$ volcanic trend can be recognized. The cones generally consist of irregular shaped vesicular bombs, scoria, and lapilli, with less common spatter and agglutinated spatter; spindle bombs are rare.

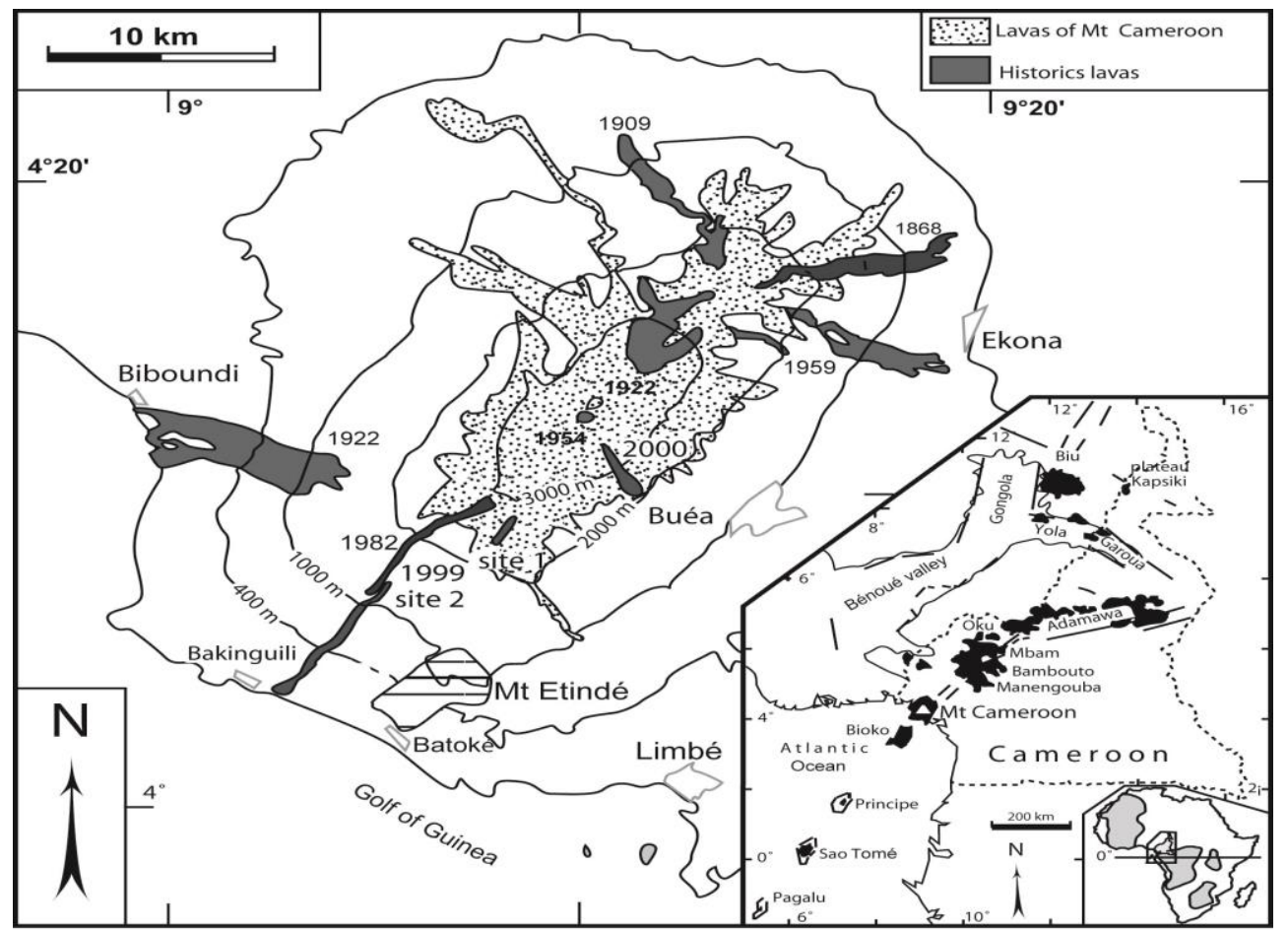

Fig. 1: Map of Mt Cameroon Showing Sample Locations; Alitudes and Rainfalls. Inset: Map Showing the Location, of the Cameroon Line in West Africa (From Déruelle Et Al. 1991). The Main Cenozoic Volcanic Centers Are Shown in Black.

The rock associated with the scoria cones vary from 4 to $10 \mathrm{~m}$ thick and are generally composed of aa lavas, some pahoehoe near their vents. Some flows that are entirely composed of pahoehoe are only 1 to 4 m thick. Six massive lavas erupted in 1909, 1922, 1959 1982, 1999 and 2000 are selected and located as follow:

The 1909 lava flow was sampled on the north flank of Mt Cameroon at an altitude of $2300 \mathrm{~m}$. The 1922 lava flow is located on the west flank of the volcano at sea level near Bibundi town. The 1959 lava flow was sampled on the northeast flank of the volcano at an altitude of $485 \mathrm{~m}$ near Ekona. The 1982 samples were collected at mid-slope $(2400 \mathrm{~m})$, on the southwest flank of the massif. Basaltic lavas from the 1999 eruption were sampled at an altitude of $2700 \mathrm{~m}$ near the 1982 crater.

Well-defined lineaments have been reported on the geological map. The morphology of Mt Cameroon is directly related to the regional tectonic. Its structure is typically that of a horst covered with volcanic products. Recent eruptions have been produced preferentially on the SW, WSW, W and NE flanks which are $\mathrm{N} 40^{\circ} \mathrm{E}-\mathrm{N} 50^{\circ} \mathrm{E}$ trending affecting its entire basement, and with normal faults $\mathrm{N} 120-\mathrm{N} 130^{\circ} \mathrm{E}$ (Déruelle et al. 1987). These main directions, which are also formerly recognized in the basin of Mamfe (Moreau et al. 1987), correspond to crust discontinuities inherited from the Pan-African orogeny and reactivated during Cenozoic to Recent times.

\section{Sampling and analytical methods}

The main mineral phases of the Mt Cameroon basaltic rocks have been analyzed by CAMEBAX electron microprobe at Université Pierre et Marie Curie, Paris.

- clinopyroxene (iron recalculated after Droop (1987) and classification after Morimoto (1989); $15 \mathrm{kV}, 40 \mathrm{nA}, 20 \mathrm{~s}$ by element except Ti: $30 \mathrm{~s}$ ); - amphibole (iron recalculated after Leake et al. 1997; $15 \mathrm{kV}, 40 \mathrm{nA}, 15 \mathrm{~s}$ by element except $\mathrm{Ca}$ and Ti: $20 \mathrm{~s}, \mathrm{Fe}$ and $\mathrm{Mn}: 25 \mathrm{~s}$, and $\mathrm{F}$ and $\mathrm{Cl}: 30 \mathrm{~s}$ ); — Ti-magnetite and ilmenite (iron recalculated after Stormer (1983); $20 \mathrm{kV}, 40 \mathrm{nA}, 40 \mathrm{~s}$ by element except $\mathrm{Al}$ and $\mathrm{Cr}: 30 \mathrm{~s}$ ). The program of correction is from "PAP" (Pouchou and Pichoir 1991).

Whole-rock chemical analyses of basaltic rocks from Mt Cameroon were carried out at CRPG laboratory, Nancy (France). Major elements were analyzed by ICP-AES and trace elements by ICPMS. The samples were previously selected in order to limit superficial contamination, then crushed. Details of other analytical processes were presented elsewhere (Carignan et al. 2001). Samples for $\mathrm{Sr}$ and $\mathrm{Nd}$ isotopic analyses were dissolved in mixed $\mathrm{HF}-$ HNO3 (10:1) acid mixture; chemical separation was carried out by cation exchange chromatography; blanks were $<1 \mathrm{ng}$. Sr and $\mathrm{Nd}$ isotopic ratios were measured on a VG Sector 54 multicollector 
thermal ionisation mass spectrometer ("Université Libre de Bruxelles"). Replicate analyses of the MERCK Nd standard gave an average ${ }^{143} \mathrm{Nd}={ }^{144} \mathrm{Nd}$ value of 0.5127428 (normalized to ${ }^{143} \mathrm{Nd}={ }^{144} \mathrm{Nd}^{1} / 40.7219$ ), and measurements of NBS $987 \mathrm{Sr}$ yielded an average ${ }^{87} \mathrm{Sr}={ }^{86} \mathrm{Sr}$ value of 0.710247 (normalized to ${ }^{86} \mathrm{Sr}={ }^{88} \mathrm{Sr}^{1 / 40.1194)}$. Epsilon $\mathrm{Nd}$ values were calculated assuming ${ }^{147} \mathrm{Sm}={ }^{144} \mathrm{Nd}^{1} / 40.1967$ and ${ }^{143} \mathrm{Nd}={ }^{144} \mathrm{Nd}^{1} / 40.512638$ for CHUR (see Ashwal et al. 2002 for a detailed description of the procedure). CIPW normative compositions were calculated on a waterfree basis with $\mathrm{Fe}_{2} \mathrm{O}_{3} / \mathrm{FeO}=0.2$ for basalt and 0.3 for hawaiite according to Middlemost (1989).

\section{Nomenclature and petrography}

The Mt Cameroon rocks are named according to their differentiation index (Thornton and Tuttle, 1960). Alkali basalts $10<$ D.I. < 35: (s.1); $35<$ D.I. < 50: hawaiites. Mg rich basalts are corresponding to those with the $\mathrm{MgO}$ contents higher than $12 \mathrm{wt} \%$ and $\mathrm{Mg} \#>50, \mathrm{Mg} \#=100 *\left[\mathrm{Mg} /\left(\mathrm{Mg}+\mathrm{Fe}^{2+}\right)\right]$.

Mg-rich basalt $(10.8<$ D.I. < 19.7) are usually massive with variable aMts of vesicles. Vesicles are more abundant in the scoriaceous samples, and they have a microlitic porphyric texture with abundant (30-40\% vol.), olivine phenocrysts $(0.7-3 \mathrm{~mm})$ and $\mathrm{Ca}$ rich pyroxene (15-25\% vol., 1-2 mm) and spinel, magnetite, and anhedral strain-twinned Ca-rich pyroxene xenocrysts. The matrix is composed of olivine and Ca-rich pyroxene $(<0.3 \mathrm{~mm})$, plagioclase (bytownite-labradorite) microlites, amphibole (brown hornblende) and glass.

Basalts $(20<$ D.I. $<35)$ have a porphyric microlitic texture; some rare samples are less porphyric. Olivine (8-10\% vol) and Ca-rich pyroxene $(3-4 \%)$ phenocrysts are present in various proportions. The matrix contains fine plagioclase microlites, oriented or not, numerous $\mathrm{Fe}-\mathrm{Ti}$ oxides and small grains of $\mathrm{Ca}$-rich pyroxene. All phenocrysts seem to be fresh and, particularly, there is no iddingsite in the olivine cracks. Plagioclase crystals are euhedral to subhedral, and usually contain glassy inclusion-rich zones

Hawaiites $(35.4$ < D.I. < 46.2) contain abundant plagioclase phenocrysts $(20-15$ vol \%), subordinate olivine phenocrysts and strongly zoned Ca-rich pyroxene phenocrysts with pink core and brownish rim in a fine-grained groundmass rich in plagioclase, granular pyroxene, and equant opaque oxides $(10-30 \mu \mathrm{m})$.

\section{Mineralogy}

\subsection{Olivine}

Mg-rich olivine phenocryts (Table 1) occurs in olivine basalts, very rich olivine basalts, and hawaiites. In basalts, the crystals are generally homogeneous, but can sometimes have Mg-rich cores (the most magnesian ones occurring in the very rich olivine basalts. The phenocrysts are usually unzoned Fo86-81) and less magnesian rims (Fo78). In hawaiites, the crystals are also generally homogeneous, but can sometimes have Mg-rich cores (the most magnesian ones occurring in the hawaiite $\mathrm{C} 8 \mathrm{C}$ ). The phenocrysts are usually unzoned Fo79-73) and less magnesian rims (Fo62). Fig. 2 shows that, with the exception of some magnesian cores of xenocrysts from hawaiite $\mathrm{C} 1 \mathrm{~W}$, the compositions of olivine plot on a well-defined trend of increasing $\mathrm{Fe}$ and $\mathrm{Mn}$ contents on the $\mathrm{Mg}$ $\mathrm{Fe}-\mathrm{Mn}$ diagram. Their $\mathrm{CaO}$ content is low $(<0.5$ wt. \%), and suggests low-pressure crystallization (Simkin and Smith, 1970). Furthermore, the relatively homogeneous distribution of Ca from cores to rims of phenocrysts may indicate the dominance of a cooling trend with little change in pressure which is rather low (Stormer, 1973).

Table 1: Representative Chemical Analyses of Olivine from Mt Cameroon Rocks

\begin{tabular}{|c|c|c|c|c|c|c|c|c|c|c|c|c|c|c|c|c|c|c|c|}
\hline Rock type Sample Description & $\begin{array}{l}\text { basalt } \\
\text { C10R } \\
\text { ph. c }\end{array}$ & ph.c & ph.c & ph. c & ph. c & $\begin{array}{l}\text { C5B } \\
\text { ph. c }\end{array}$ & $\begin{array}{l}\text { C8B } \\
\text { ph. c }\end{array}$ & $\begin{array}{l}\text { C9P } \\
\text { ph. c }\end{array}$ & $\begin{array}{l}00-5 \\
\text { ph. c }\end{array}$ & ph. r & $\begin{array}{l}00-3 \\
\text { ph. c }\end{array}$ & $\begin{array}{l}\text { 00-4 } \\
\text { ph. c }\end{array}$ & $\begin{array}{l}99-02 \\
\text { ph. c }\end{array}$ & $\begin{array}{l}\text { hawaiite } \\
\text { C8C } \\
\text { ph. c }\end{array}$ & $\begin{array}{l}\text { C8D } \\
\text { ph. c }\end{array}$ & $\begin{array}{l}\text { C1W } \\
\text { x. c } \\
\end{array}$ & $\begin{array}{l}\text { C10D } \\
\text { ph. c }\end{array}$ & $\begin{array}{l}00-1 \\
\text { ph. c }\end{array}$ & ph. \\
\hline $\mathrm{SiO}_{2}$ (\% wt.) & 39.59 & 39.48 & 39.57 & 39.35 & \multirow{2}{*}{39.5614 .30} & 39.82 & 39.73 & 39.87 & 39.37 & 38.20 & 39.11 & 39.35 & 40.06 & 39.28 & 39.40 & 40.67 & 37.51 & 37.17 & 34.99 \\
\hline $\mathrm{FeO}$ & 13.80 & 13.85 & 14.65 & 14.28 & & 16.74 & 15.16 & 14.02 & 15.72 & 22.62 & 16.03 & 16.74 & 15.19 & 19.71 & 20.33 & 11.18 & 24.03 & 33.20 & 35.08 \\
\hline $\mathrm{MnO}$ & 0.15 & 0.28 & 0.20 & 0.26 & 0.20 & 0.27 & 0.21 & 0.11 & 0.25 & 0.40 & 0.25 & 0.22 & 0.19 & 0.36 & 0.35 & 0.10 & 0.47 & 1.03 & 1.05 \\
\hline $\mathrm{MgO}$ & 45.10 & 45.35 & 45.36 & 44.81 & 45.62 & 42.46 & 43.37 & 45.46 & 44.15 & 38.35 & 43.70 & 42.94 & 43.58 & 40.25 & 39.94 & 47.48 & 36.80 & 29.13 & 29.05 \\
\hline $\mathrm{CaO}$ & 0.32 & 0.33 & 0.31 & 0.40 & 0.34 & 0.30 & 0.36 & 0.33 & 0.32 & 0.29 & 0.30 & 0.31 & 0.26 & 0.33 & 0.42 & 0.12 & 0.41 & 0.37 & 0.38 \\
\hline $\mathrm{NiO}$ & 0.22 & 0.22 & 0.13 & 0.19 & 0.20 & 0.21 & 0.20 & 0.22 & 0.13 & 0.04 & 0.24 & 0.14 & 0.00 & 0.13 & 0.12 & 0.41 & 0.06 & 0.05 & 0.01 \\
\hline Total & 99.17 & 99.50 & 100.22 & 99.28 & 100.22 & 99.80 & 99.03 & 100.01 & 99.94 & 99.90 & 99.63 & 99.70 & 99.28 & 100.06 & 100.56 & 99.96 & 99.28 & 100.95 & 100.56 \\
\hline Si (a.p.f.u) & 0.999 & 0.994 & 0.992 & 0.995 & 0.990 & 1.009 & 1.012 & 0.997 & 0.993 & 0.997 & 0.991 & 1.001 & 1.017 & 1.011 & 1.012 & 1.006 & 0.993 & 1.017 & 0.964 \\
\hline $\mathrm{Fe}$ & 0.291 & 0.292 & 0.307 & 0.302 & 0.299 & 0.357 & 0.323 & 0.287 & 0.317 & 0.487 & 0.322 & 0.356 & 0.322 & 0.424 & 0.437 & 0.231 & 0.517 & 0.759 & 0.735 \\
\hline $\mathrm{Mn}$ & 0.003 & 0.006 & 0.004 & 0.006 & 0.004 & 0.006 & 0.005 & 0.002 & 0.005 & 0.009 & 0.005 & 0.005 & 0.004 & 0.008 & 0.008 & 0.002 & 0.011 & 0.024 & 0.025 \\
\hline $\mathrm{Mg}$ & 1.696 & 1.702 & 1.695 & 1.689 & 1.703 & 1.616 & 1.647 & 1.695 & 1.659 & 1.492 & 1.651 & 1.628 & 1.649 & 1.545 & 1.530 & 1.750 & 1.452 & 1.188 & 1.193 \\
\hline $\mathrm{Ca}$ & 0.009 & 0.009 & 0.008 & 0.011 & 0.009 & 0.008 & 0.010 & 0.009 & 0.009 & 0.008 & 0.008 & 0.008 & 0.007 & 0.009 & 0.012 & 0.003 & 0.012 & 0.011 & 0.011 \\
\hline $\mathrm{Ni}$ & 0.004 & 0.005 & 0.003 & 0.004 & 0.004 & 0.004 & 0.004 & 0.004 & 0.003 & 0.001 & 0.005 & 0.003 & 0.000 & 0.003 & 0.003 & 0.008 & 0.001 & 0.001 & 0.000 \\
\hline Fo(\%) & 85.2 & 85.1 & 84.5 & 84.6 & 84.9 & 81.9 & 83.6 & 85.5 & 84.0 & 75.4 & 83.7 & 82.1 & 83.7 & 78.5 & 77.8 & 88.3 & 73.7 & 61.0 & 61.9 \\
\hline $\mathrm{Fa}$ & 14.8 & 14.9 & 15.5 & 15.4 & 15.1 & 18.1 & 16.4 & 14.5 & 16.0 & 24.6 & 16.3 & 17.9 & 16.4 & 21.6 & 22.2 & 11.7 & 26.3 & 39.0 & 38.1 \\
\hline Mg\# & 85.35 & 85.37 & 84.66 & 84.83 & 85.05 & 81.89 & 83.60 & 85.52 & 83.98 & 75.38 & 83.68 & 82.05 & 83.64 & 78.45 & 77.79 & 88.33 & 73.73 & 61.00 & 61.87 \\
\hline
\end{tabular}




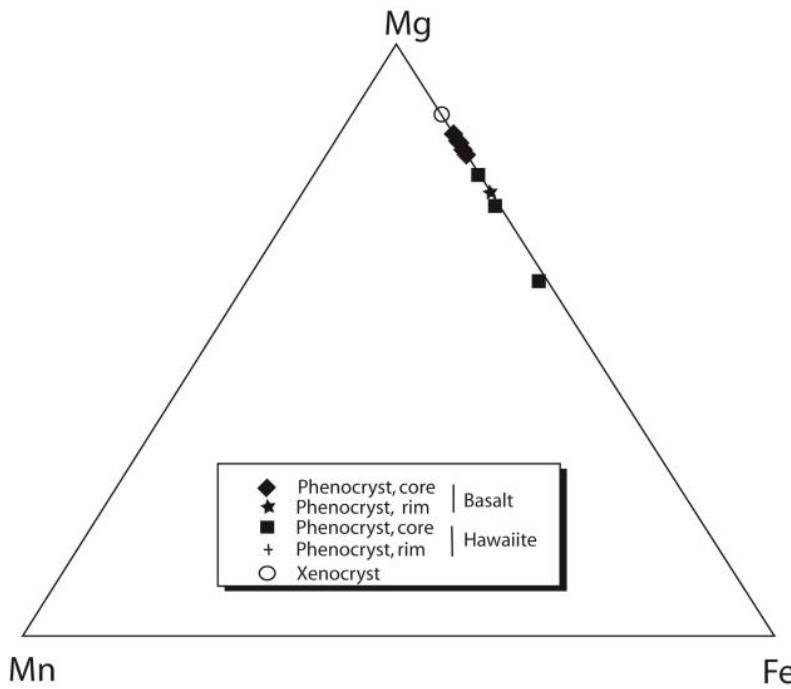

Fig. 2: Distribution of the Olivine Compositions in the Mg-Fe-Mn Diagram for the Volcanic Rocks of Mt Cameroon.

Olivine phenocrysts in the host lavas crystallized most probably during and after eruption to the surface because of expansion of the olivine stability field at shallower pressure. This is consistent with calculated olivine/whole-rock temperatures of $1200^{\circ} \mathrm{C}(-$ 40/+20) (Puturika, 1997). Equilibrium between olivine and wholerock was assumed if calculated equilibrium olivine compositions $\left(\right.$ with $\mathrm{KD}_{\mathrm{D}}{ }^{\mathrm{ol} / \mathrm{liq}}(\mathrm{Mg}-\mathrm{Fe})=0.30 \pm 0.003$, Roeder and Emslie $(1970)$ matched measured olivine phenocryst cores.

\subsection{Ca-rich pyroxene}

Ca-rich pyroxene is present in basalts and hawaiites (Table 2). Its composition ranges from Wo48 En 46 Fs6 to Wo45 En43 Fs12 and belongs to the diopside field (Morimoto et al. 1988) of the pyroxene quadrilateral. The phenocrysts are rich in aluminium $(3.4<$ $\left.\mathrm{Al}_{2} \mathrm{O}_{3}<7.5 \%\right)$ and titanium $\left(1.7<\mathrm{TiO}_{2}<3.5 \%\right)$, the highest $\mathrm{Al}$ and $\mathrm{Ti}$ contents being found in rims (Fig. 3). Calculated $\mathrm{Al}^{\mathrm{VI}} / \mathrm{Al}^{\mathrm{IV}}$ ratios have a larger range in cores $(0.18-0.72)$ than in rims $(0.13-0.46)$. Ti/Al ratios range from 0.11 to 0.29 in cores and from 0.17 to 0.37 in rims. All these data indicate that the cores have equilibrated as relatively low pressures (Wass, 1979). The systematically low $\mathrm{Na}$ when compared with the high aMt of $\mathrm{Al}$ and $\mathrm{Ti}$ and noticeable $\mathrm{Fe}^{3+}$ estimated contents, indicate that $\mathrm{Ca}-\mathrm{Ti}-$ Tschermak's and Ca-Tschermak's molecules are the other major components.

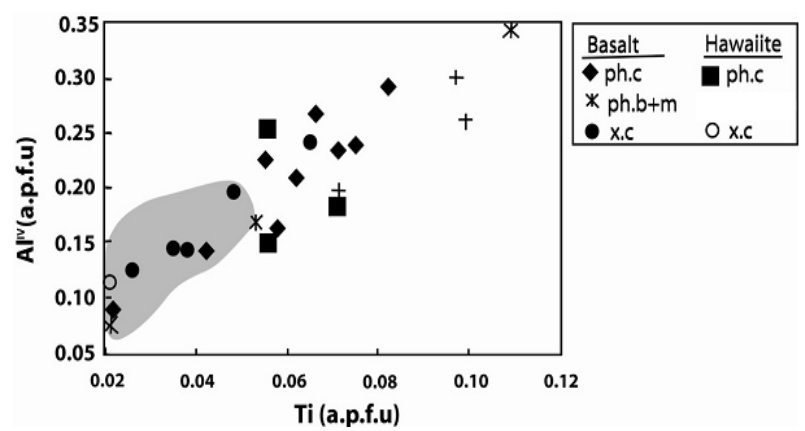

Fig. 3: Compositional Variations of Clinopyroxene Phenocrysts, Xenocrysts and Microlites from the Mt Cameroon Volcanic Rocks. Data of Xenolith of Mt Cameroon are from Ngounouno Et Al (2001).

The microlites of Ca-rich pyroxene (Wo45 $\mathrm{En}_{45} \mathrm{Fs}_{10}$ ) contain less $\mathrm{Al}^{\mathrm{VI}}$ than the phenocrysts, showing to their low-pressure crystallization. Assuming a temperature of $1000^{\circ} \mathrm{C}$, equilibrium pressures could be estimated at $0.3 \pm 0.04 \mathrm{GPa}$ (after Nimis, 1998). Clinopyroxene compositions of the lavas are quite similar to those of the xenoliths from the mantle. Clinopyroxene phenocryst rims and microlites have high $\mathrm{Al}_{2} \mathrm{O}_{3}$ contents (4.5-6.1 wt. \%).

Table 2: Representative Chemical Analyses of Clinopyroxene from Mt Cameroon Rocks

\begin{tabular}{|c|c|c|c|c|c|c|c|c|c|c|c|c|c|c|c|c|c|c|c|c|c|c|c|c|c|c|}
\hline Rock type & basalt & & & & & & & & & & & & & & & & & hawaiite & & & & & & & & \\
\hline $\begin{array}{l}\text { Sample } \\
\text { Description }\end{array}$ & $\begin{array}{l}\text { C10R } \\
\text { ph. c }\end{array}$ & ph.b & ph. c & $\begin{array}{l}\text { C5B } \\
\text { ph. c }\end{array}$ & x. c & $\begin{array}{l}\mathrm{C} 8 \mathrm{~B} \\
\text { x. c }\end{array}$ & $\begin{array}{l}\text { C9P } \\
\text { ph. c }\end{array}$ & $\begin{array}{l}00-5 \\
\text { ph. c }\end{array}$ & x. c & $\begin{array}{l}00-3 \\
\text { ph. c }\end{array}$ & x. c & $\begin{array}{l}00-4 \\
\text { ph. c }\end{array}$ & $\mathrm{m}$ & X. c & $\begin{array}{l}\text { C10W } \\
\text { ph. c }\end{array}$ & $\begin{array}{r}\mathrm{C} 8 \mathrm{C} \\
\text { ph. c }\end{array}$ & ph. b & $\begin{array}{l}\text { C8D } \\
\text { ph. c }\end{array}$ & a. b & $\begin{array}{l}\text { C1W } \\
\text { ph. c }\end{array}$ & ph. b & $\begin{array}{l}\text { C10D } \\
\text { x. c }\end{array}$ & $\begin{array}{l}00-1 \\
\text { ph.b }\end{array}$ & $\begin{array}{l}\text { C10F } \\
\text { ph. c }\end{array}$ & h. b & $\begin{array}{l}00-6 \\
\text { ph. c }\end{array}$ \\
\hline & 52.28 & 52.31 & 47.53 & 49.18 & 49.94 & 47.07 & 49.84 & 45.64 & 50.02 & 47.03 & 50.78 & 47.16 & 49.37 & 48.47 & 48.18 & 48.84 & 47.98 & 47.30 & 46.33 & 48.11 & 45.47 & 51.84 & 50.49 & 49.94 & 44.08 & 46.97 \\
\hline $\mathrm{TiO} 2$ & 0.77 & 0.77 & 1.96 & 2.05 & 1.37 & 2.31 & 1.50 & 2.93 & 1.24 & 2.66 & 0.95 & 2.51 & 1.89 & 1.70 & 2.21 & 2.55 & 2.52 & 2.00 & 3.51 & 2.27 & 3.46 & 0.82 & 1.78 & 2.00 & 3.84 & 2.35 \\
\hline $\mathrm{Al} 2 \mathrm{O} 3$ & 2 & 2.51 & 6.22 & 4.09 & 3. & 6.18 & 3.81 & 7.69 & 4.22 & 6.23 & 3. & .37 & 4.53 & 5.52 & 5.29 & 5.19 & 5.41 & 6.16 & 6.47 & .14 & 7.45 & 2.24 & 2.85 & 4.47 & 8.13 & 6.89 \\
\hline $\mathrm{Cr} 2 \mathrm{O} 3$ & 0. & 0.41 & 46 & 01 & 32 & 43 & 00 & 04 & 0.63 & 0.03 & 0.92 & 0.02 & 0.0 & 0.63 & .00 & 0.01 & 0.0 & 0.00 & 0.0 & .00 & 0.00 & 0.73 & 0.00 & .01 & .00 & 0.04 \\
\hline & & & & 39 & & 55 & & & & & & & & & & 7. & & 8.3 & & 7.27 & 8.06 & 5. & 7.68 & 86 & 9.10 & 8.15 \\
\hline M & & 0.04 & 2 & 18 & 13 & 0.11 & 0 & 0.15 & 0.08 & & & 0.12 & 0.14 & 0 & 0.07 & 0.18 & 0.20 & 0.14 & 0.40 & 0.13 & 0.14 & 0.07 & 0.28 & .21 & 0.15 & 0.15 \\
\hline $\mathrm{MgO}$ & 15.88 & 15.96 & 13.14 & 3.87 & 14.60 & 3.01 & 14.17 & 12.76 & 14.83 & 12.89 & 15.37 & 12.79 & 14.05 & 13.82 & 13.28 & 13.34 & 12.92 & 14.06 & 11.68 & 3.41 & 12.09 & 15.78 & 14.08 & 13.69 & 11.48 & 12.60 \\
\hline $\mathrm{CaO}$ & 23.06 & 22.60 & 22.53 & 2.58 & 22.74 & 2.66 & 21.15 & 21.07 & 22.51 & 22.34 & 22.29 & 22.40 & 22.37 & 22.58 & 22.76 & 22.32 & 22.26 & 21.98 & 21.85 & 22.59 & 22.78 & 22.45 & 21.84 & 22.43 & 22.52 & 22.62 \\
\hline $\mathrm{Na}$ & 0. & 0.39 & 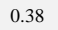 & 40 & ? & 25 & ( & 0.64 & 0.33 & 44 & 0.3 & 0.4 & 0.38 & 0.39 & 0.39 & 0. & 0.47 & 0.33 & 0. & 0.42 & 0.4 & 0.32 & 0.51 & 0.4 & 49 & 0.35 \\
\hline Total & 100.3 & 99.49 & 99.47 & 99.25 & 99.34 & 99.67 & 99.49 & 99.42 & 99.31 & 99.29 & 99.53 & 99.39 & 99.87 & 99.45 & 100.06 & 99.92 & 99.01 & 100.27 & 100.11 & 99.34 & 99.89 & 99.97 & 99.51 & 100.09 & 99.79 & 100.12 \\
\hline $\mathrm{Si}$ (a.r & 1.909 & 1.925 & 1.773 & .837 & 1.854 & 1.757 & 1.855 & 1.706 & 1.854 & 1.761 & 1.875 & 1.764 & 1.831 & 1.802 & 1.790 & 1.816 & 1.802 & 1.748 & 1.739 & 1.797 & 1.699 & 1.922 & .884 & .850 & 1.656 & 1.733 \\
\hline $\mathrm{Ti}$ & 21 & 0.021 & 055 & 058 & 0.038 & 665 & 0.042 & 32 & 5 & 75 & 26 & 0.071 & 53 & 0 & 0 & 0 & 0.071 & 56 & 0.099 & 0.064 & 0.097 & 0.023 & .050 & 56 & 09 & 0.066 \\
\hline AIIV & 91 & 0.075 & .227 & 163 & 0.146 & 243 & 0.145 & 0.294 & 0.146 & 0.239 & 0.126 & 0.236 & 0.169 & 0.198 & 0.2 & 0.184 & 0.198 & 0.253 & 0.261 & 0.203 & 0.300 & 0.078 & 0.117 & 0.150 & 0.344 & 0.268 \\
\hline AIVI & 30 & 333 & 0.047 & 17 & 225 & 28 & 22 & 0.045 & 00 & 36 & 0 & 45 & 029 & 44 & 0.02 & 3 & 0.042 & 0.0 & 0.025 & 0.024 & 0.028 & 0.020 & 0.009 & .047 & .016 & 0.037 \\
\hline CI & 0.009 & 0.012 & 0.014 & 000 & 0.009 & 0.013 & 0.000 & 0.001 & 0.019 & 0.001 & 0.027 & 0.001 & 0.000 & 0.019 & 0.000 & 0.000 & 0.000 & 0.000 & 0.000 & 0.000 & 0.000 & 0.029 & 0.000 & . .000 & 0.000 & 0.001 \\
\hline $\mathrm{Fe} 3+$ & 0.036 & 0.015 & 0.084 & .060 & 0.059 & 0.098 & 0.079 & 0.130 & 0.044 & 0.084 & 0.034 & 0.080 & 0.062 & 0.069 & 0.093 & 0.031 & 0.048 & 0.149 & 0.079 & 0.082 & 0.109 & 0.014 & .045 & 0.029 & .147 & 0.123 \\
\hline $\mathrm{Fe} 2+$ & & 0.124 & 138 & 155 & 0.128 & 138 & 0.181 & 0.136 & 0.125 & 54 & 0.123 & 0.158 & 0.159 & 0.126 & 0. & 0.188 & 0.179 & 0.107 & 0.220 & 0.145 & 0.143 & 0.132 & 195 & 0.184 & 0.139 & 0.132 \\
\hline $\mathrm{Mn}$ & & & & & & & & & & & & & & & & & & & & & & 0.002 & .009 & 07 & 05 & 0.005 \\
\hline $\mathrm{Mg}$ & 0 & 0 & 0 & 72 & 0.808 & 0.724 & 0. & 0. & 0. & 20 & 16 & 13 & 0 & 0.766 & 0 & 0. & 3 & 0.7 & 0.6 & 0.747 & 0.674 & 0.872 & .783 & 56 & 43 & 0.703 \\
\hline $\mathrm{Ca}$ & 0.902 & 0.891 & 0.900 & 903 & 0.905 & 0.906 & 0.843 & 0.814 & 0.894 & 0.896 & 0.882 & 0.898 & 0.889 & 99 & 0.906 & 0.889 & 896 & 70 & 0.879 & 0.904 & 0.912 & 0.892 & .873 & .890 & .906 & 0.907 \\
\hline $\mathrm{Na}$ & 0.026 & 0.028 & 0.027 & .029 & 0.024 & 0.025 & 0.042 & 0.046 & 0.024 & 0.032 & 24 & 0.031 & 0.027 & 0.028 & 0.028 & 0.032 & 0.034 & 0.024 & 0.036 & 0.030 & 0.032 & 0.023 & .037 & .035 & .036 & 0.025 \\
\hline Wo & 46.19 & 45.72 & 46.11 & 6.28 & 46.24 & 46.41 & 43.53 & 43.18 & 45.64 & 45.90 & 45.05 & 45.92 & 45.30 & 46.16 & 46.44 & 45.37 & 45.88 & 44.02 & 44.88 & 46.40 & 46.93 & 45.35 & 44.96 & 5.63 & 6.9 & 45.85 \\
\hline En & & 47. & 46. & 11 & 46.83 & .00 & 46. & 48.89 & 47.39 & 45.23 & 88 & 44.91 & 45.83 & 46 & & & 43 & 50.90 & 41 & 45.53 & 44.84 & 47.67 & 4.15 & 72 & 27 & 45.11 \\
\hline Fs & & & & & & & & & & & & & & & & & $.5 \%$ & & & & 8.21 & 6.96 & 0.88 & 0.64 & .831 & 03 \\
\hline $\mathrm{Mg}$ \# & 88.58 & 87.61 & 84.10 & 83.28 & 86.32 & 84.03 & 81.28 & 83.94 & 86.76 & 82.37 & 87.30 & 81.90 & 83.01 & 85.84 & 82.88 & 79.71 & 80.13 & 87.86 & 74.82 & 83.74 & 82.49 & 86.85 & 80.06 & 80.43 & 82.23 & 84.15 \\
\hline
\end{tabular}
$\mathrm{ph}=$ phenocryst, $\mathrm{c}=$ core, $\mathrm{b}=$ rim, $\mathrm{x}=$ xenocryst, $\mathrm{m}=$ microlite, $\mathrm{a} . \mathrm{p}$. $\mathrm{f}$. $\mathrm{u}=$ Atome Per Formula Unit. 


\subsection{Amphibole}

Compositions of Ca-amphibole microphenocrysts $(<0.5 \mathrm{~mm})$ vary between $\mathrm{Mg}^{\#}$ : 49 in some olivine basalts, very rich olivine basalts and hawaiites (1999-2) (Table 3). Ca-amphibole is relatively rich in $\mathrm{Ti}(0.22 \mathrm{Ti}$ atoms/23 oxygen), classifying the mineral as kaersutite hornblende (Fig. 4) (after Leake et al. 1997). The presence of Ca-amphibole microphenocrysts in some very rich olivine basalts indicates that the Mt Cameroon magma had a relatively high $\mathrm{P}_{\mathrm{H} 2 \mathrm{O}}$, a feature that is also a characteristic of the Cameroon Hot Line basaltic magmas (Ngounouno et al. 2005). The melt from which these basalts formed was probably enriched in volatiles during crystallization of early basalts.

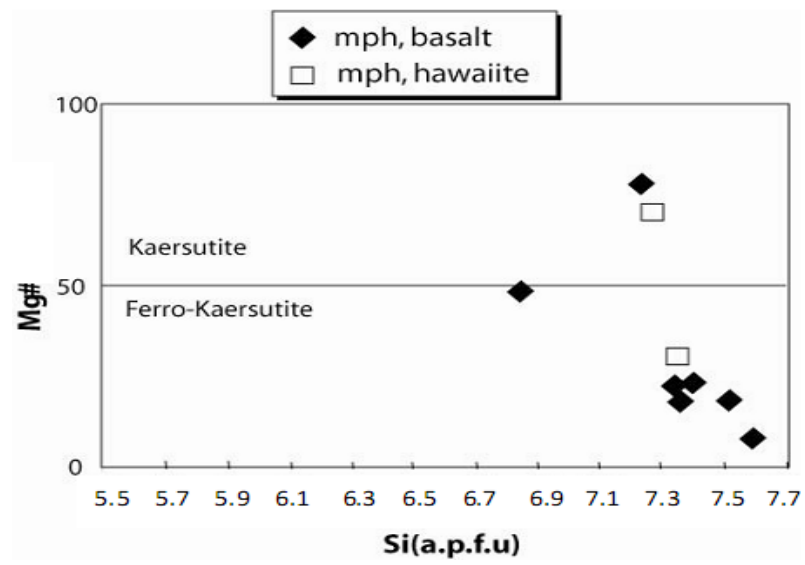

Fig. 4: Compositional Variations of Amphibole Microphenocrysts from the Mt Cameroon Volcanic Rocks in Mg\# vs. Si Diagram (After Leake Et Al. 1998)

Table 3: Representative Chemical Analyses of Microphenocryst of Amphibole from Mt Cameroon Rocks

\begin{tabular}{|c|c|c|c|c|c|c|c|}
\hline Rock type & basalt & & & & & hawaiite & \\
\hline Sample & C10R & & $\mathrm{C} 10 \mathrm{~J}$ & $99-02$ & & $99-3$ & $00-1$ \\
\hline Description & mph. c & mph. c & mph. c & mph. c & mph. c & mph. c & mph. c \\
\hline $\mathrm{SiO} 2$ (wt. \%) & 48.90 & 45.89 & 48.90 & 48.37 & 49.57 & 45.34 & 50.93 \\
\hline $\mathrm{TiO} 2$ & 3.34 & 1.98 & 3.89 & 2.98 & 2.83 & 4.60 & 0.60 \\
\hline $\mathrm{Al} 2 \mathrm{O} 3$ & 16.07 & 13.81 & 17.01 & 15.86 & 16.54 & 11.70 & 6.28 \\
\hline $\mathrm{FeO}^{*}$ & 11.31 & 14.23 & 9.86 & 8.43 & 8.64 & 7.82 & 10.96 \\
\hline $\mathrm{MnO}$ & 0.21 & 0.23 & 0.22 & 0.18 & 0.14 & 0.23 & 0.33 \\
\hline $\mathrm{MgO}$ & 4.31 & 7.58 & 2.27 & 6.04 & 4.78 & 6.98 & 15.55 \\
\hline $\mathrm{Na} 2 \mathrm{O}$ & 2.41 & 2.95 & 3.42 & 2.87 & 3.75 & 1.56 & 1.08 \\
\hline $\mathrm{K} 2 \mathrm{O}$ & 2.02 & 2.78 & 2.20 & 1.92 & 2.90 & 1.48 & 0.22 \\
\hline Total & 99.13 & 95.80 & 99.10 & 99.64 & 99.95 & 95.89 & 97.74 \\
\hline Si (a.p.f.u) & 7.356 & 6.853 & 7.595 & 7.355 & 7.522 & 7.393 & 7.243 \\
\hline $\mathrm{Ti}$ & 0.377 & 0.222 & 0.455 & 0.340 & 0.323 & 0.564 & 0.064 \\
\hline Al IV & 0.644 & 1.147 & 0.405 & 0.645 & 0.478 & 0.607 & 0.757 \\
\hline $\mathrm{Al} \mathrm{VI}$ & 2.206 & 1.284 & 2.709 & 2.197 & 2.481 & 1.641 & 0.296 \\
\hline $\mathrm{Fe} 3+$ & 0.000 & 0.002 & 0.000 & 0.000 & 0.000 & 0.000 & 0.402 \\
\hline $\mathrm{Mn}$ & 0.027 & 0.029 & 0.029 & 0.023 & 0.018 & 0.032 & 0.040 \\
\hline $\mathrm{Mg}$ & 0.967 & 1.688 & 0.527 & 1.368 & 1.081 & 1.697 & 3.297 \\
\hline $\mathrm{Ca}$ & 1.704 & 1.016 & 1.884 & 2.119 & 1.755 & 2.827 & 1.796 \\
\hline $\mathrm{Na}$ & 0.702 & 0.854 & 1.031 & 0.845 & 1.105 & 0.493 & 0.298 \\
\hline $\mathrm{K}$ & 0.365 & 0.530 & 0.397 & 0.344 & 0.518 & 0.280 & 0.040 \\
\hline $\mathrm{mg \#}$ & 18.6 & 49.0 & 8.4 & 22.3 & 18.0 & 23.0 & 79.0 \\
\hline
\end{tabular}

$\mathrm{mph}=$ microphenocryst, $\mathrm{c}=$ core.

\subsection{Plagioclase}

Plagioclase compositions are highly calcic $\left(\mathrm{An}_{87}-\mathrm{An}_{58}\right)$, from basalts to hawaiites (Table 4). They are rich in $\mathrm{SrO}$ (up to $0.67 \mathrm{wt}$. $\%)$. Iron is present in significant aMts $\left(\mathrm{Fe}_{2} \mathrm{O}_{3}\right.$ : up to $\left.1.5 \%\right)$. Plagioclase phenocryts are normally zoned in basalts with bytownite $\left(A n_{73-71}\right)$ cores and oligoclase $\left(\mathrm{An}_{18}\right)$ rims. Labradorite $\left(\mathrm{An}_{74-}\right.$
An55) microlites occurs in the groundmass. Basalt $\mathrm{C} 10 \mathrm{~W}$ contains plagioclase compositions which range from $\mathrm{An}_{71}$ to $\mathrm{An}_{18}$ with ternary composition $\left(\mathrm{An}_{71-18} \mathrm{Ab}_{37-2} \mathrm{Or}_{79-2}\right)$, and plagioclase microlites $\left(\mathrm{An}_{55}\right)$. Hawaiites contain plagioclase phenocrysts $(\approx 40 \%$ vol.) whose composition range from $\mathrm{An}_{81}$ to $\mathrm{An}_{61}$ and microlites $(0.5 \times 0.1 \mathrm{~mm})$ from $\mathrm{An}_{87}$ to $\mathrm{An}_{57}$.

Table 4: Representative Chemical Analyses of Plagioclase from Mt Cameroon Rocks

\begin{tabular}{|c|c|c|c|c|c|c|c|c|c|c|c|c|c|c|c|}
\hline $\begin{array}{l}\text { Rock tvne } \\
\text { Sample }\end{array}$ & $\begin{array}{l}\text { hasalt } \\
\text { C5B }\end{array}$ & $99-3$ & & & $00-5$ & & $00-3$ & $00-4$ & & $\mathrm{C} 10 \mathrm{~W}$ & & & & $00-6$ & $\mathrm{C} 8 \mathrm{D}$ \\
\hline Descrintion & & nh. c & nh. $r$ & $\mathrm{~m}$ & nh. c & nh. $\mathrm{r}$ & nh. c & nh. c & $\mathrm{m}$ & ph. c & nh. $r$ & $\mathrm{~m}$ & $\mathrm{~m}$ & nh. c & $\mathrm{m}$ \\
\hline $\mathrm{SiO} 2$ (wt.\%) & 50.87 & 52.85 & 52.18 & 51.83 & 51.53 & 55.60 & 48.30 & 51.17 & 52.86 & 50.69 & 50.87 & 48.84 & 51.11 & 54.71 & 51.56 \\
\hline $\mathrm{Al} 2 \mathrm{O} 3$ & 30.54 & 29.12 & 29.62 & 29.76 & 29.75 & 27.80 & 32.52 & 30.21 & 29.01 & 30.25 & 30.41 & 30.98 & 31.12 & 28.26 & 30.46 \\
\hline $\mathrm{CaO}$ & 13.50 & 12.17 & 12.73 & 13.06 & 13.12 & 9.84 & 15.97 & 13.38 & 12.36 & 14.84 & 14.01 & 14.38 & 3.17 & 10.84 & 13.46 \\
\hline $\mathrm{Na} 2 \mathrm{O}$ & 3.52 & 4.18 & 3.88 & 3.73 & 3.58 & 5.18 & 2.14 & 3.51 & 4.02 & 3.12 & 3.44 & 3.34 & 0.19 & 4.67 & 3.49 \\
\hline $\mathrm{K} 2 \mathrm{O}$ & 0.25 & 0.30 & 0.24 & 0.30 & 0.29 & 0.62 & 0.01 & 0.23 & 0.35 & 0.16 & 0.22 & 0.25 & 13.94 & 0.41 & 0.36 \\
\hline $\mathrm{SrO}$ & 0.53 & 0.00 & 0.00 & 0.00 & 0.00 & 0.00 & 0.00 & 0.00 & 0.00 & 0.25 & 0.48 & 0.37 & 0.12 & 0.00 & 0.27 \\
\hline $\mathrm{BaO}$ & 0.25 & 0.00 & 0.00 & 0.00 & 0.00 & 0.00 & 0.00 & 0.00 & 0.00 & 0.03 & 0.11 & 0.25 & 0.00 & 0.00 & 0.17 \\
\hline Total & 100.33 & 99.29 & 99.41 & 99.48 & 99.01 & 99.74 & 99.57 & 99.22 & 99.40 & 100.14 & 100.35 & 99.59 & 100.69 & 99.58 & 100.66 \\
\hline Si (a.p.f.u) & 2.32 & 2.42 & 2.39 & 2.37 & 2.37 & 2.53 & 2.22 & 2.35 & 2.42 & 2.30 & 2.34 & 2.28 & 2.39 & 2.49 & 2.34 \\
\hline $\mathrm{Al}$ & 1.64 & 1.57 & 1.60 & 1.61 & 1.61 & 1.45 & 1.76 & 1.64 & 1.56 & 1.67 & 1.62 & 1.67 & 1.72 & 1.51 & 1.63 \\
\hline $\mathrm{Fe} 3+$ & 0.03 & 0.00 & 0.00 & 0.00 & 0.00 & 0.00 & 0.00 & 0.00 & 0.00 & 0.03 & 0.01 & 0.05 & 0.04 & 0.00 & 0.02 \\
\hline $\mathrm{Fe} 2+$ & 0.00 & 0.03 & 0.03 & 0.00 & 0.03 & 0.03 & 0.02 & 0.03 & 0.03 & 0.00 & 0.02 & 0.00 & 0.00 & 0.03 & 0.02 \\
\hline $\mathrm{Ca}$ & 0.66 & 0.60 & 0.62 & 0.64 & 0.70 & 0.48 & 0.79 & 0.66 & 0.61 & 0.72 & 0.68 & 0.70 & 0.16 & 0.53 & 0.66 \\
\hline $\mathrm{Na}$ & 0.31 & 0.37 & 0.35 & 0.33 & 0.32 & 0.45 & 0.19 & 0.31 & 0.36 & 0.28 & 0.30 & 0.30 & 0.02 & 0.41 & 0.31 \\
\hline $\mathrm{K}$ & 0.02 & 0.02 & 0.01 & 0.02 & 0.02 & 0.04 & 0.00 & 0.01 & 0.02 & 0.01 & 0.01 & 0.03 & 0.82 & 0.02 & 0.02 \\
\hline $\mathrm{Sr}$ & 0.01 & 0.00 & 0.00 & 0.00 & 0.00 & 0.00 & 0.00 & 0.00 & 0.00 & 0.01 & 0.01 & 0.01 & 0.00 & 0.00 & 0.01 \\
\hline
\end{tabular}




\begin{tabular}{|c|c|c|c|c|c|c|c|c|c|c|c|c|c|c|c|}
\hline An $(\%)$ & 66.90 & 60.50 & 63.50 & 64.70 & 66.70 & 50.60 & 80.40 & 67.70 & 62.70 & 71.70 & 68.00 & 69.20 & 15.80 & 55.90 & 66.50 \\
\hline $\mathrm{Ab}$ & 32.00 & 37.70 & 35.10 & 33.50 & 31.60 & 45.80 & 19.60 & 31.00 & 35.30 & 28.00 & 30.60 & 30.30 & 2.00 & 41.70 & 31.50 \\
\hline Or & 1.10 & 1.80 & 1.40 & 1.80 & 1.70 & 3.60 & 0.00 & 1.30 & 2.00 & 0.30 & 1.40 & 0.50 & 82.20 & 2.40 & 3.00 \\
\hline
\end{tabular}

\begin{tabular}{|c|c|c|c|c|c|c|c|c|c|c|c|c|c|}
\hline $\begin{array}{l}\text { Rock tvne } \\
\text { Sample }\end{array}$ & $\begin{array}{l}\text { hawaiit } \\
\text { C1W }\end{array}$ & & & $99-2$ & & $00-1$ & & $\mathrm{C} 10 \mathrm{~F}$ & & & $\mathrm{C} 8 \mathrm{C}$ & & \\
\hline Descrintion & nh c & nh b & $\mathrm{m}$ & nh. c & nh. $b$ & nh. c & $\mathrm{m}$ & nh. c & wh. $b$ & $\mathrm{~m}$ & wh. c & wh. $b$ & $\mathrm{~m}$ \\
\hline $\mathrm{SiO} 2$ (\% wt.) & 48.23 & 49.55 & 52.70 & 54.50 & 53.42 & 48.81 & 53.98 & 48.87 & 52.94 & 52.55 & 47.40 & 49.22 & 53.56 \\
\hline $\mathrm{A} 12 \mathrm{O} 3$ & 33.39 & 32.72 & 29.90 & 28.19 & 28.82 & 31.64 & 28.00 & 33.09 & 29.86 & 29.61 & 33.52 & 32.59 & 29.00 \\
\hline $\mathrm{CaO}$ & 15.63 & 14.60 & 12.84 & 11.18 & 11.77 & 15.29 & 11.00 & 15.01 & 12.67 & 12.17 & 16.28 & 15.31 & 12.03 \\
\hline $\mathrm{Na} 2 \mathrm{O}$ & 1.99 & 2.50 & 4.04 & 4.89 & 4.42 & 2.61 & 4.99 & 2.50 & 4.27 & 4.38 & 1.98 & 2.52 & 4.22 \\
\hline $\mathrm{K} 2 \mathrm{O}$ & 0.07 & 0.17 & 0.23 & 0.40 & 0.33 & 0.12 & 0.44 & 0.10 & 0.30 & 0.48 & 0.12 & 0.13 & 0.36 \\
\hline $\mathrm{SrO}$ & 0.37 & 0.48 & 0.29 & 0.00 & 0.00 & 0.00 & 0.00 & 0.30 & 0.28 & 0.33 & 0.25 & 0.42 & 0.28 \\
\hline $\mathrm{BaO}$ & 0.25 & 0.00 & 0.00 & 0.00 & 0.00 & 0.00 & 0.00 & 0.00 & 0.00 & 0.17 & 0.31 & 0.00 & 0.08 \\
\hline Total & 100. & 100.88 & 100.82 & 99.87 & 99.48 & 99.13 & 99.10 & 100.51 & 101.15 & 100.87 & 100.25 & 100.92 & 100.45 \\
\hline Si (a.p.f.u) & 2.20 & 2.25 & 2.38 & 2.47 & 2.44 & 2.25 & 2.46 & 2.23 & 2.38 & 2.38 & 2.18 & 2.24 & 2.43 \\
\hline $\mathrm{Al}$ & 1.80 & 1.75 & 1.59 & 1.51 & 1.55 & 1.72 & 1.51 & 1.78 & 1.58 & 1.58 & 1.81 & 1.75 & 1.55 \\
\hline $\mathrm{Fe} 3+$ & 0.00 & 0.00 & 0.02 & 0.01 & 0.00 & 0.01 & 0.03 & 0.00 & 0.03 & 0.00 & 0.02 & 0.01 & 0.00 \\
\hline $\mathrm{Fe} 2+$ & 0.02 & 0.03 & 0.02 & 0.02 & 0.03 & 0.02 & 0.00 & 0.02 & 0.00 & 0.00 & 0.00 & 0.02 & 0.04 \\
\hline $\mathrm{Ca}$ & 0.77 & 0.71 & 0.62 & 0.54 & 0.58 & 0.76 & 0.54 & 0.73 & 0.61 & 0.59 & 0.80 & 0.75 & 0.58 \\
\hline $\mathrm{Na}$ & 0.18 & 0.22 & 0.36 & 0.43 & 0.39 & 0.23 & 0.44 & 0.22 & 0.37 & 0.39 & 0.18 & 0.22 & 0.37 \\
\hline $\mathrm{K}$ & 0.00 & 0.01 & 0.01 & 0.02 & 0.02 & 0.01 & 0.03 & 0.01 & 0.02 & 0.03 & 0.01 & 0.01 & 0.02 \\
\hline $\mathrm{Sr}$ & 0.01 & 0.01 & 0.01 & 0.00 & 0.00 & 0.00 & 0.00 & 0.01 & 0.01 & 0.01 & 0.01 & 0.01 & 0.01 \\
\hline $\mathrm{Ba}$ & 0.00 & 0.00 & 0.00 & 0.00 & 0.00 & 0.00 & 0.00 & 0.00 & 0.00 & 0.00 & 0.01 & 0.00 & 0.00 \\
\hline Or & 0.90 & 0.00 & 1.00 & 2.30 & 1.90 & 0.00 & 2.60 & 0.00 & 1.00 & 2.00 & 0.00 & 0.00 & 0.00 \\
\hline
\end{tabular}

$\mathrm{ph}=$ phenocryst, $\mathrm{c}=$ core $, \mathrm{r}=\mathrm{rim}, \mathrm{m}=$ microlite.

\subsection{Fe-Ti oxides}

A Fe-Ti oxide occurs throughout the Mt Cameroon volcanic rocks and representative analyses are presented in (Table 5). In basalts, titanomagnetite $(46<$ Usp \% < 57) occurs as subhedral crystals ( 0.5 to $1.0 \mathrm{~mm})$ enclosed in olivine or Ti-diopside phenocrysts. In basalt, ilmenite lamellae are associated with titanomagnetite phenocrysts. Equilibrium temperatures and oxygen fugacities of coexisting magnetite and ilmenite were calculated following Spencer and Lindsley (1981) and Stormer (1973) with uncertainties of $40-80^{\circ} \mathrm{C}$ for temperatures and $0.5-1.0 \times 10^{-10}$ atm for $\mathrm{fO}_{2}$. They are around $1300^{\circ} \mathrm{C}\left( \pm 20{ }^{\circ} \mathrm{C}\right)$ and $10^{-13}\left( \pm 0.5 \times 10^{-13}\right)$ atmos- pheres. In other basalts, magnetite phenocrysts are rich in $\mathrm{Ti}(17.6$ $\% \mathrm{TiO}_{2}$ ), $\mathrm{Mg}$ (up to $8 \% \mathrm{MgO}$ ), $\mathrm{Al}\left(9.3<\mathrm{Al}_{2} \mathrm{O}_{3}<14.6 \%\right.$ ) and $\mathrm{Cr}$ $\left(5.1<\mathrm{Cr}_{2} \mathrm{O}_{3}<15.0 \%\right)$. Such Ti-Al-Cr-rich magnetites have already been observed in alkali basalts from Mururoa where their origin has been interpreted as a result of an exchange process between Cr-spinel and host-magmas (Maury et al. 1992).

Table 5: Representative Chemical Analyses of Fe-Ti Oxides from Mt Cameroon Rocks

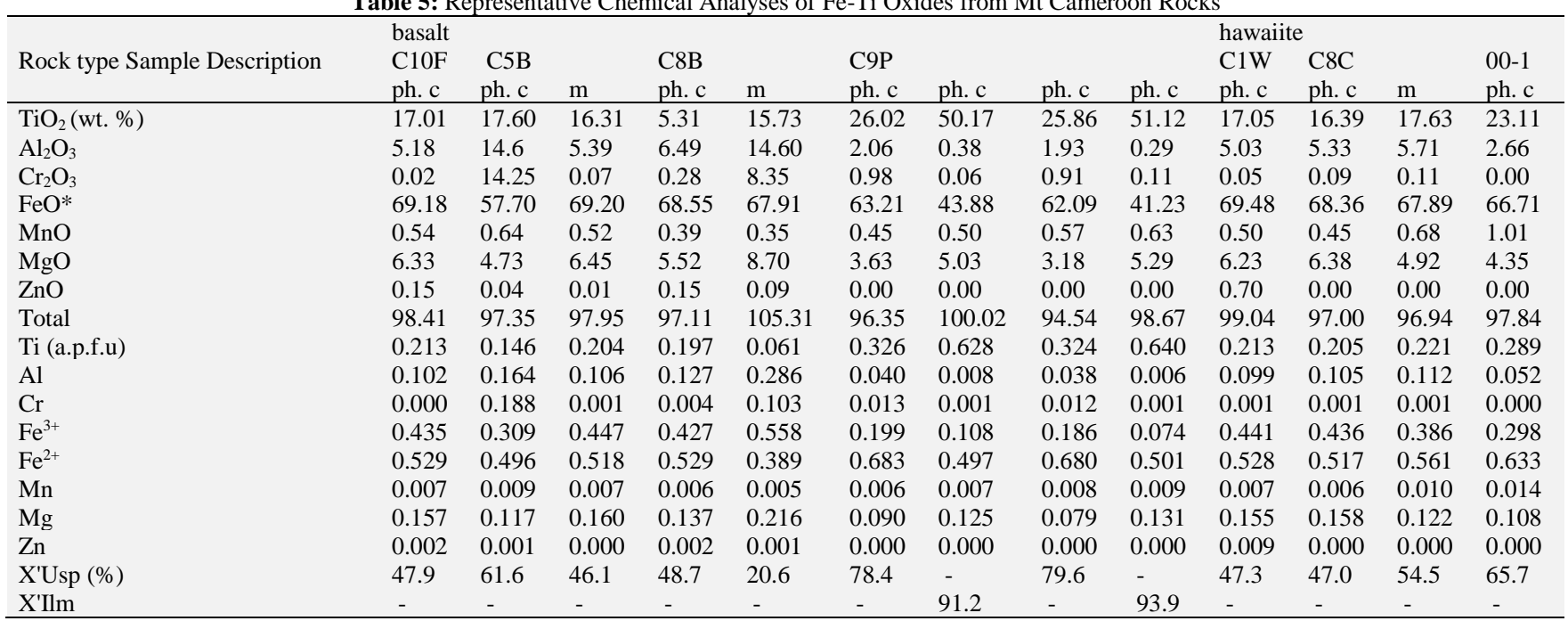

$\mathrm{ph}=$ phenocryst $\mathrm{c}=\mathrm{core} ; \mathrm{b}=$ rime $; \mathrm{x}=$ xenocryst, $\mathrm{m}=$ microlite $;$ a.p.f.u= Atome Per Formula Unit

\subsection{Xenocrysts}

The mantle assemblage is composed of forsteritic olivine, chromespinel and chrome-diopside. This assemblage had not been found complete in many single types of lava; however, the constituent minerals occur in various basalts and hawaiites. In other lava flows (C8C, C9U), the assemblage is well represented by xenoliths (Ngounouno et al. 2005). Corroded forsteritic olivine (Fo88) with an abundance of pale-brown picotite inclusions may be the distinctive characteristic of the mantle assemblage. The resorbed forteritic olivine is associated with xenocrysts of chrome-spinel and chrome-diopside.

Olivine xenocryst occurs in hawaiite $\mathrm{C} 1 \mathrm{~W}$; they are easily distinguished from phenocrysts by their rounded shape and their numerous cracks. They have higher $\mathrm{Mg \#}\left(\mathrm{Fo}_{88}\right)$ and $\mathrm{Ni}$ contents and lower $\mathrm{Mn}$ and $\mathrm{Ca}$ contents than phenocrysts (Table 1). Similar high Ni-Mg olivine xenocrysts were reported from alkali basalts from the Kapsiki Plateau (Ngounouno et al. 2000) and the Upper Benue valley (Ngounouno et al. 2003). The $\mathrm{Ni}-\mathrm{Mg}$ rich olivine described here could thus be considered as a cognate near liquidus phase of basaltic liquids more primitive than their present-day host 
hawaiite. Their crystallization temperature $\left(1400 \pm 70{ }^{\circ} \mathrm{C}\right.$, estimate as for phenocrysts, see above) and pressure (0.7 GPa, estimate after Presnall et al. 1978) correspond to those occurring at the discontinuity between the lower crust and the upper mantle $(\approx$ $30 \mathrm{~km})$.

$\mathrm{Cr}$-spinel has been observed in $\mathrm{Mg}$ rich basalts (C10R) and basalts (00-5), where it occurs as scarce small xenocysts $(0.3$ to $0.5 \mathrm{~mm})$ scattered in the groundmass and as inclusions $(<0.2 \mathrm{~mm})$ in olivine phenocrysts. Its composition is intermediate between those of chromite and magnesio-chromite (Fig. 5, Table 6). Inclusions in olivine range in $\mathrm{Cr}^{\#}\left(\mathrm{Cr}^{\#}=[\mathrm{Cr} /(\mathrm{Cr}+\mathrm{Al})]\right.$ from 15 to 44 and in $\mathrm{Mg} \#=\left[\mathrm{Mg} /\left(\mathrm{Mg}+\mathrm{Fe}^{2+}\right)\right]$ from 17 to 27 ; the Ti-content varies between 8.2 and $20.7 \mathrm{wt} \% \mathrm{TiO}_{2}$. In contrast the composition of the xenocrysts ranges from $\mathrm{Cr} \#$ 61-68 and $\mathrm{Mg} \# 48-54$ and Ticontents are $\approx 2.5 \mathrm{wt} \% \mathrm{TiO}_{2}$. The relatively high $\mathrm{Cr} \#$ in inclusions is related to the low $\mathrm{Al}$ contents (3.8-9.7 wt \% $\left.\mathrm{Al}_{2} \mathrm{O}_{3}\right)$. In fact, inclusions have lower contents of $\mathrm{Cr}\left(0.98-9.7 \mathrm{wt} \% \mathrm{Cr}_{2} \mathrm{O}_{3}\right)$ than xenocrysts $\left(38.0-43.5\right.$ wt. $\left.\% \mathrm{Cr}_{2} \mathrm{O}_{3}\right)$.

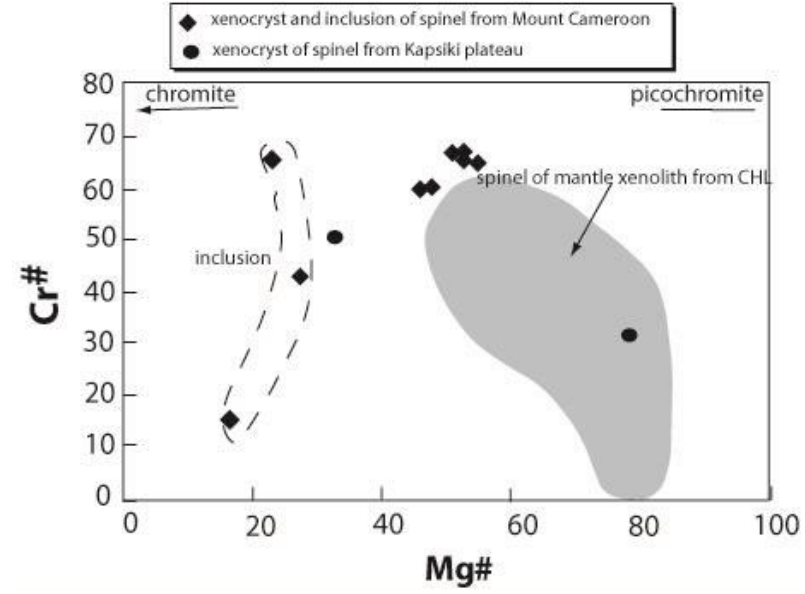

Fig. 5: Compositional Variations of Spinel Xenocrysts and Inclusion from the.

Table 6: Representative Chemical Analyses of Xenocryst and Inclusion of Spinel from Mt Cameroon Rocks

\begin{tabular}{|c|c|c|c|c|c|c|c|c|c|c|c|}
\hline $\begin{array}{l}\text { Lava type } \\
\text { Sample } \\
\text { Description }\end{array}$ & $\begin{array}{l}\text { basalt } \\
\text { C10R } \\
\text { ph. c } \\
\end{array}$ & ph. c & ph. c & ph. c & ph. c & ph. c & $\begin{array}{l}00-5 \\
\text { ph. c }\end{array}$ & ph. c & incl & incl & incl \\
\hline $\mathrm{TiO}_{2}$ (wt.\%) & 2.55 & 2.53 & 2.69 & 2.40 & 2.57 & 2.60 & 2.42 & 2.32 & 8.24 & 11.00 & 20.67 \\
\hline $\mathrm{Al}_{2} \mathrm{O}_{3}$ & 14.60 & 14.46 & 13.26 & 13.97 & 14.38 & 14.35 & 16.66 & 16.45 & 9.74 & 8.42 & 3.75 \\
\hline $\mathrm{Cr}_{2} \mathrm{O}_{3}$ & 41.53 & 42.04 & 41.77 & 43.47 & 41.45 & 41.28 & 37.57 & 38.02 & 28.40 & 9.69 & 0.98 \\
\hline $\mathrm{FeO}$ & 28.87 & 28.72 & 31.02 & 24.49 & 30.67 & 30.85 & 33.16 & 33.29 & 47.50 & 59.40 & 64.21 \\
\hline $\mathrm{MnO}$ & 0.28 & 0.23 & 0.19 & 0.21 & 0.22 & 0.25 & 0.29 & 0.30 & 0.50 & 0.36 & 0.75 \\
\hline $\mathrm{MgO}$ & 12.14 & 12.04 & 11.33 & 11.70 & 11.83 & 11.82 & 10.65 & 10.15 & 5.49 & 6.63 & 4.75 \\
\hline Total & 100.14 & 100.15 & 100.38 & 96.42 & 101.24 & 101.29 & 100.97 & 100.71 & 99.87 & 95.60 & 95.15 \\
\hline Ti (a.p.f.u) & 0.482 & 0.479 & 0.513 & 0.456 & 0.487 & 0.493 & 0.455 & 0.431 & 1.682 & 2.314 & 4.540 \\
\hline $\mathrm{Al}$ & 4.328 & 4.292 & 3.965 & 4.157 & 4.271 & 4.261 & 4.911 & 4.877 & 3.114 & 2.776 & 1.291 \\
\hline $\mathrm{Cr}$ & 8.259 & 8.372 & 8.378 & 8.677 & 8.260 & 8.223 & 7.626 & 7.562 & 6.092 & 2.143 & 0.226 \\
\hline $\mathrm{Fe}^{3+}$ & 2.447 & 2.377 & 2.630 & 2.255 & 2.495 & 2.529 & 2.553 & 2.682 & 3.431 & 6.453 & 5.403 \\
\hline $\mathrm{Fe}^{2+}$ & 3.836 & 3.883 & 4.163 & 3.971 & 3.970 & 3.971 & 4.382 & 4.532 & 7.346 & 7.442 & 0.277 \\
\hline $\mathrm{Mn}$ & 0.060 & 0.049 & 0.041 & 0.045 & 0.046 & 0.053 & 0.061 & 0.064 & 0.115 & 0.085 & 0.186 \\
\hline $\mathrm{Mg}$ & 4.553 & 4.521 & 4.285 & 4.404 & 4.446 & 4.440 & 3.967 & 3.807 & 2.221 & 2.765 & 2.068 \\
\hline $\mathrm{Cr}^{\#}$ & 65.6 & 66.1 & 67.9 & 67.6 & 65.9 & 65.9 & 60.5 & 60.8 & 66.2 & 43.6 & 14.9 \\
\hline $\mathrm{mg}^{\#}$ & 54.3 & 53.8 & 50.7 & 52.6 & 52.8 & 52.8 & 47.5 & 45.7 & 23.2 & 27.1 & 16.8 \\
\hline
\end{tabular}

$\mathrm{ph}=$ phenocryst $; \mathrm{c}=$ core; incl $=$ inclusion.

Mt Cameroon volcanic rock in Cr\# vs Mg\# diagram. Data of xenolith are from Lee et al. (1994); Ngounouno et al. (2001); Temdjim et al. (2005).

Rounded and fracturated Cr-diopside xenocrysts are present in some basalts. They have a fairly restricted composition range (see Fig. 3), with low $\mathrm{Al}$, high $\mathrm{Mg \#} \mathrm{(0.85-0.92)} \mathrm{and} \mathrm{Cr}$ contents (up to

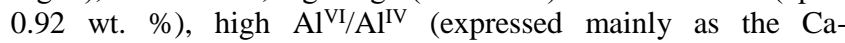
Tschermak molecule), and moderate $\mathrm{Na}_{2} \mathrm{O}$ contents $(0.20$ to 0.69 wt. \%) expressed as the jadeite molecule.

\section{Whole-rock geochemistry}

\subsection{Major and trace elements variation}

Major and trace elements compositions of representative samples of the Mt Cameroon lavas series are presented in Table 7. All the rocks are nepheline-normative (1.9\% to $17.1 \%$ ) alkaline basaltic rocks. $\mathrm{SiO}_{2}$ contents range from 43 wt $\%$ to 47 wt $\%$ which indicated a low degree of crystallization of the Mt Cameroon volcanic series. Mg-rich basalts have primitive characteristics (MgO: 12.9-15.0 wt \%, Cr: 360-205 ppm, Cr: 650-1300 ppm) and all the other lavas are slightly evolved. Their primitive nature is confirmed by the relatively low D.I. (10.8-24.0).

Throughout the entire series, $\mathrm{TiO}_{2}$ concentrations vary from less than $1.5 \mathrm{wt} \%$ to almost $4 \mathrm{wt} \%$. The low $\mathrm{TiO}_{2}$ abundances are in rocks dominated by plagioclase, olivine, and $\mathrm{Ca}$-rich pyroxene, while those with high abundances are enriched in kaersutite, Tirich spinel, Ti-magnetite and ilmenite. The hawaiites have high potassium abundances (1.6-1.8 wt \%)

$\mathrm{Ni}$ and $\mathrm{Cr}$ contents are significantly high in Mg-rich basalts (sample C10J contains Ni up to $279 \mathrm{ppm}$ and $\mathrm{Cr}$ up to $1304 \mathrm{ppm}$ ), attesting to the primitive nature of this lava. The $\mathrm{CaO} / \mathrm{Al}_{2} \mathrm{O}_{3}$ ratio is low (0.6-1.8) and decreases with decreasing $\mathrm{MgO}$.

Table 7: Major and Trace Elements for Mt Cameroon Rocks

\begin{tabular}{|c|c|c|c|c|c|c|c|c|c|c|c|c|c|c|c|c|c|c|c|c|c|c|c|c|}
\hline Lava type & basalt & & & & & & & & & & & & & & & & & & & & & & & \\
\hline Sample & $\mathrm{C} 10 \mathrm{~J}$ & C5 M & C9 U & C8 A & С5B 1922 & С8B 1868 & $\mathrm{C} 8 \mathrm{H}$ & C8 P & C8 Q & C11 C & C5 P & C9 T2 & C10 D & C8 D & C5 S & $\mathrm{C} 5 \mathrm{H}$ & C5U & C10R & $\begin{array}{l}00-5 \\
2000\end{array}$ & C5 N & C9 P & $\begin{array}{l}00-6 \\
2000\end{array}$ & $\begin{array}{l}99-03 \\
1999\end{array}$ & $\mathrm{C} 8 \mathrm{~F}$ \\
\hline $\mathrm{SiO}_{2}$ & 45.00 & 45.24 & 43.20 & 45.00 & & 44.87 & 44.38 & 44.91 & 44.56 & 44.92 & ? & & & & 43.0 & & & & 44.69 & & 47.11 & 45.55 & 45.44 & 44.88 \\
\hline $\mathrm{TiO}_{2}$ & 2 . & 49 & 2.83 & 2.69 & & & 2.77 & 2.8 & 2.90 & 2.8 & 2.9 & 3. & 5 & 3.4 & 3. & 3.2 & 2.89 & 2. & 3.09 & 2.3 & 3.0 & 3.09 & 3.06 & .87 \\
\hline $\mathrm{Al}_{2} \mathrm{O}_{3}$ & 8.43 & .53 & 9.87 & .24 & 2.00 & .68 & 3.09 & 12.71 & 12.68 & 3.21 & 3.43 & 13.40 & 4.22 & 14.72 & 15.43 & 14.36 & 15.20 & 15.01 & 14.96 & 14.06 & 14.80 & 15.47 & 15.65 & 15.47 \\
\hline $\mathrm{Fe}_{2} \mathrm{O}_{3}$ & 11.07 & 2.78 & 13.79 & 12.74 & 13.48 & 12.82 & 13.22 & 12.86 & 12.92 & 13.09 & 13.17 & 13.43 & 12.77 & 13.46 & 13.39 & 13.24 & 12.22 & 12.04 & 12.53 & 12.08 & 11.65 & 12.41 & 12.58 & 11.90 \\
\hline $\mathrm{MnO}$ & 0.18 & 17 & 0.17 & 0.18 & 0.20 & 0.18 & 0.18 & 0.18 & 0.19 & 0.19 & 0.18 & 0.17 & 0.17 & 0.20 & 0.20 & 0.20 & 0.19 & 0.19 & 0.19 & 0.18 & 0.15 & 0.19 & 0.19 & 0.19 \\
\hline $\mathrm{MgO}$ & 14.46 & 13.95 & 12.92 & 10.79 & 10.71 & 10.04 & 9.91 & 9.39 & 9.35 & 9.30 & 8.89 & 7.55 & 7.19 & 6.85 & 6.34 & 7.48 & 7.04 & 7.17 & 7.04 & 6.99 & 6.81 & 6.72 & 6.49 & 6.29 \\
\hline
\end{tabular}




\begin{tabular}{|c|c|c|c|c|c|c|c|c|c|c|c|c|c|c|c|c|c|c|c|c|c|c|c|c|}
\hline $\mathrm{CaO}$ & 14.57 & 10.60 & 14.07 & 12.79 & 11.72 & 12.60 & 13.04 & 13.19 & 12.96 & 11.61 & 12.49 & 11.93 & 10.75 & 12.07 & 11.80 & 11.35 & 11.12 & 11.46 & 11.07 & 11.19 & 10.55 & 10.89 & 10.56 & 10.85 \\
\hline $\mathrm{Na}_{2} \mathrm{O}$ & 1.26 & 2.20 & 1.35 & 2.48 & 3.21 & 2.65 & 2.43 & 2.72 & 2.28 & 2.91 & 2.27 & 3.12 & 2.62 & 3.73 & 2.89 & 3.91 & 3.86 & 3.28 & 3.81 & 4.10 & 3.07 & 4.00 & 4.06 & 3.86 \\
\hline $\mathrm{K}_{2} \mathrm{O}$ & 0.59 & 0.79 & 0.37 & 0.85 & 1.34 & 0.89 & 0.88 & 1.02 & 1.07 & 1.06 & 0.80 & 1.33 & 1.09 & 1.63 & 1.00 & 1.58 & 1.26 & 1.30 & 1.46 & 1.32 & 1.30 & 1.59 & 1.59 & 1.76 \\
\hline $\mathrm{P}_{2} \mathrm{O}_{5}$ & 0.32 & 0.42 & 0.41 & 0.46 & 0.74 & 0.47 & 0.42 & 0.49 & 0.48 & 0.52 & 0.53 & 0.67 & 0.55 & 0.81 & 0.71 & 0.81 & 0.54 & 0.45 & 0.55 & 0.49 & 0.52 & 0.62 & 0.61 & 0.70 \\
\hline L.O.I & 1.87 & -0.34 & 0.71 & -0.56 & -0.34 & -0.48 & -0.36 & -0.51 & 0.00 & 0.00 & 0.92 & 0.03 & 1.05 & -0.40 & 1.40 & 0.03 & -0.50 & -0.26 & -0.47 & -0.13 & 0.71 & -0.55 & -0.19 & 0.73 \\
\hline Total & 100.02 & 99.83 & 99.69 & 99.66 & 99.06 & 99.46 & 99.96 & 99.80 & 99.39 & 99.69 & 98.99 & 99.89 & 99.77 & 99.75 & 99.65 & 99.58 & 99.98 & 99.16 & 98.92 & 98.84 & 99.72 & 99.98 & 100.04 & 99.50 \\
\hline $\mathrm{Be}(\mathrm{ppm})$ & $\operatorname{tr}$ & 0.97 & 0.92 & 0.92 & 1.72 & 0.96 & 0.95 & 0.95 & 0.72 & 1.19 & 1.20 & 1.54 & 1.28 & 2.07 & 1.50 & 1.57 & 1.77 & 1.63 & 1.78 & 2.62 & 1.47 & 1.81 & 2.29 & 1.95 \\
\hline $\mathrm{Rb}$ & 14.3 & 17.9 & 6.6 & 19.2 & 32.0 & 21.5 & 20.8 & 22.4 & 24.4 & 25.3 & 19.6 & 33.2 & 24.8 & 39.9 & 24.7 & 41.8 & 35.8 & 30.6 & 35.4 & 46.2 & 33.0 & 35.9 & 37 & 46.8 \\
\hline $\mathrm{Sr}$ & 473 & 583 & 561 & 686 & 926 & 720 & 737 & 671 & 715 & 752 & 833 & 861 & 710 & 1167 & 1064 & 1143 & 1062 & 860 & 981 & 1174 & 776 & 998 & 1019 & 1055 \\
\hline Cs & 0.27 & 0.24 & 0.07 & 0.21 & 0.34 & 0.19 & 0.29 & 0.25 & 0.37 & 0.33 & 0.25 & 0.35 & 0.14 & 0.54 & 0.35 & 0.41 & 0.36 & 0.37 & 0.41 & 0.64 & 0.21 & 0.46 & 0.66 & 0.63 \\
\hline $\mathrm{Ba}$ & 205 & 275 & 236 & 258 & 392 & 259 & 276 & 290 & 310 & 331 & 336 & 440 & 361 & 545 & 449 & 492 & 450 & 372 & 433 & 604 & 383 & 453 & 475 & 556 \\
\hline V & 235 & 241 & 304 & 281 & 249 & 287 & 316 & 281 & 325 & 291 & 292 & 295 & 274 & 332 & 299 & 261 & 283 & 266 & 293 & 245 & 298 & 286 & 260 & 263 \\
\hline $\mathrm{Cr}$ & 1304 & 952 & 642 & 572 & 491 & 566 & 453 & 388 & 518 & 406 & 307 & 270 & 286 & 173 & 94.1 & 160 & 206 & 262 & 218 & 153 & 272 & 181 & 141 & 189 \\
\hline Co & 57 & 60 & 61 & 51 & 46 & 52 & 53 & 44 & 50 & 49 & 46 & 44 & 42 & 43 & 37 & 37 & 41 & 43 & 44 & 39 & 37 & 41 & 39 & 37 \\
\hline $\mathrm{Ni}$ & 279 & 367 & 268 & 216 & 181 & 205 & 204 & 130 & 158 & 185 & 132 & 120 & 122 & 90 & 60 & 92 & 102 & 98 & 92 & 94 & 93 & 79 & 82 & 77 \\
\hline $\mathrm{Cu}$ & 55 & 82 & 94 & 97 & 57 & 104 & 153 & 88 & 117 & 101 & 98 & 96 & 124 & 120 & 60 & 58 & 96 & 75 & 82 & 88 & 37 & 74 & 81 & 81 \\
\hline $\mathrm{Zn}$ & 88 & 100 & 102 & 94 & 97 & 101 & 106 & 93 & 102 & 110 & 97 & 126 & 109 & 126 & 111 & 106 & 110 & 122 & 125 & 108 & 116 & 123 & 130 & 118 \\
\hline $\mathrm{Ga}$ & 14.0 & 17.8 & 17.1 & 18.9 & 19.5 & 20.7 & 20.0 & 17.6 & 20.0 & 21.0 & 19.6 & 23.0 & 23.4 & 24.5 & 22.4 & 23.0 & 24.5 & 21.7 & 23.6 & 23.3 & 24.7 & 23.8 & 23.90 & 23.0 \\
\hline $\mathrm{Ge}$ & 1.62 & 1.13 & 1.27 & 1.22 & 1.12 & 1.18 & 1.26 & 1.10 & 1.21 & 1.21 & 1.24 & 1.19 & 1.06 & 1.20 & 1.16 & 1.06 & 1.22 & 1.34 & 1.43 & 1.17 & 1.17 & 1.28 & 0.98 & 1.24 \\
\hline As & 0.00 & 0.49 & 0.40 & 0.69 & 0.99 & 0.76 & 0.64 & 0.74 & 0.72 & 0.76 & 0.85 & 0.91 & 0.81 & 1.37 & 0.96 & 1.14 & 1.14 & 1.08 & 1.17 & 1.74 & 0.52 & 1.18 & 1.03 & 1.34 \\
\hline $\mathrm{Cd}$ & $\operatorname{tr}$ & 0.16 & $\operatorname{tr}$ & 0.01 & 0.03 & 0.12 & tr & tr & tr & 8.83 & 0.09 & 0.12 & $\operatorname{tr}$ & 0.08 & 0.13 & tr & 0.14 & tr & tr & 0.14 & tr & tr & 0.50 & tr \\
\hline In & 0.08 & 0.11 & 0.15 & 0.06 & 0.06 & 0.06 & 0.05 & 0.06 & 0.06 & 0.10 & 0.06 & 0.16 & 0.12 & 0.08 & 0.07 & 0.06 & 0.06 & 0.11 & 0.10 & 0.07 & 0.15 & 0.10 & 0.10 & 0.13 \\
\hline $\mathrm{Sn}$ & 1.37 & 1.51 & 1.52 & 1.52 & 1.63 & 1.59 & 1.48 & 1.47 & 1.39 & 1.92 & 1.67 & 2.16 & 2.14 & 2.56 & 1.88 & 1.84 & 2.00 & 2.01 & 2.08 & 2.10 & 2.14 & 2.09 & 2.24 & 2.05 \\
\hline $\mathrm{Sb}$ & $\operatorname{tr}$ & 0.07 & 0.02 & 0.03 & tr & 0.01 & tr & 0.01 & 0.00 & 0.18 & 0.01 & 0.05 & $\operatorname{tr}$ & 0.26 & 0.07 & tr & 0.09 & tr & tr & 0.19 & 0.05 & tr & 0.13 & 0.15 \\
\hline Y & 18.3 & 20.1 & 20.9 & 22.1 & 26.1 & 24.0 & 22.5 & 20.8 & 24.0 & 25.8 & 23.9 & 27.0 & 48.8 & 31.7 & 28.6 & 29.6 & 31.1 & 25.9 & 29.2 & 32.4 & 30.2 & 30.3 & 31.4 & 28.5 \\
\hline $\mathrm{Zr}$ & 183 & 202 & 208 & 223 & 313 & 241 & 227 & 226 & 241 & 266 & 254 & 300 & 302 & 357 & 295 & 372 & 360 & 307 & 324 & 449 & 314 & 343 & 357 & 354 \\
\hline $\mathrm{Nb}$ & 39.0 & 42.6 & 42.3 & 49.3 & 80.5 & 54.1 & 53.4 & 54.4 & 61.5 & 64.4 & 64.5 & 68.7 & 59.7 & 102.2 & 78.2 & 100.0 & 88.4 & 74.2 & 82.6 & 116.2 & 58.5 & 86.9 & 87.9 & 100.2 \\
\hline Mo & 1.39 & 0.88 & 1.10 & 1.69 & 2.34 & 1.83 & 1.65 & 1.87 & 1.57 & 2.29 & 1.68 & 2.08 & 1.57 & 3.56 & 1.95 & 2.71 & 2.59 & 3.56 & 4.01 & 3.99 & 1.96 & 4.09 & 3.71 & 3.10 \\
\hline $\mathrm{Hf}$ & 4.45 & 4.51 & 5.07 & 4.96 & 7.00 & 5.32 & 5.33 & 4.98 & 5.76 & 5.71 & 5.79 & 6.88 & 7.13 & 7.93 & 6.79 & 8.47 & 8.05 & 6.66 & 7.27 & 9.83 & 7.48 & 7.41 & 7.45 & 7.72 \\
\hline Ta & 2.88 & 3.06 & 2.98 & 3.60 & 5.57 & 3.79 & 3.75 & 3.89 & 4.40 & 4.43 & 4.66 & 4.83 & 4.31 & 7.43 & 5.75 & 6.82 & 6.38 & 5.44 & 6.01 & 8.47 & 4.38 & 6.27 & 6.27 & 7.10 \\
\hline W & $\operatorname{tr}$ & 0.24 & 0.28 & 0.59 & 0.78 & 0.61 & 0.51 & 0.67 & 0.66 & 0.86 & 0.69 & 0.55 & 0.46 & 1.32 & 0.96 & 0.63 & 0.94 & 1.00 & 1.19 & 1.27 & 0.57 & 1.22 & 1.25 & 1.10 \\
\hline $\mathrm{Pb}$ & 1.95 & 2.44 & 1.70 & 2.04 & 2.86 & 2.16 & 1.96 & 2.05 & 3.30 & 2.17 & 2.73 & 3.48 & 2.27 & 3.69 & 3.11 & 3.98 & 4.05 & 3.65 & 3.86 & 5.86 & 5.78 & 3.86 & 4.19 & 4.08 \\
\hline $\mathrm{Bi}$ & $\mathrm{tr}$ & tr & $\operatorname{tr}$ & $\operatorname{tr}$ & $\operatorname{tr}$ & $\operatorname{tr}$ & $\operatorname{tr}$ & $\operatorname{tr}$ & $\operatorname{tr}$ & 0.06 & $\operatorname{tr}$ & 0.02 & $\operatorname{tr}$ & 0.07 & $\operatorname{tr}$ & $\operatorname{tr}$ & $\operatorname{tr}$ & 0.06 & $\operatorname{tr}$ & $\operatorname{tr}$ & 0.00 & $\mathrm{tr}$ & 0.02 & 0.00 \\
\hline Th & 3.26 & 3.39 & 3.36 & 3.57 & 6.47 & 3.90 & 3.94 & 4.11 & 4.64 & 4.71 & 5.33 & 5.57 & 4.22 & 9.16 & 6.37 & 8.76 & 8.01 & 6.70 & 7.72 & 11.93 & 7.16 & 7.89 & 8.98 & 8.33 \\
\hline $\mathrm{U}$ & 0.88 & 0.92 & 0.63 & 1.06 & 1.62 & 1.10 & 1.00 & 1.20 & 1.24 & 1.36 & 1.46 & 1.40 & 0.88 & 2.51 & 1.65 & 2.16 & 2.13 & 1.85 & 1.97 & 3.36 & 1.32 & 2.04 & 2.02 & 2.15 \\
\hline $\mathrm{La}$ & 32.7 & 33.3 & 36.8 & 39.2 & 69.7 & 42.4 & 43.6 & 43.0 & 50.1 & 51.3 & 53.2 & 55.9 & 61.0 & 86.0 & 64.9 & 88.2 & 74.0 & 61.4 & 69.6 & 96.5 & 50.4 & 72.1 & 75.9 & 78.9 \\
\hline $\mathrm{Ce}$ & 70 & 70 & 76 & 85 & 137 & 94 & 92 & 94 & 103 & 108 & 113 & 11 & 105 & 160 & 133 & 168 & 146 & 123 & 139 & 183 & 106 & 143 & 153 & 156 \\
\hline $\operatorname{Pr}$ & 8.7 & 8.5 & 9.2 & 10.4 & 15.3 & 10.6 & 10.5 & 10.8 & 12.9 & 12.3 & 12.8 & 13.7 & 16.7 & 19.4 & 15.5 & 18.1 & 16.5 & 14.4 & 16.1 & 20.5 & 13.0 & 16.7 & 17.7 & 16.4 \\
\hline $\mathrm{Nd}$ & 35.1 & 32.6 & 36.4 & 42.2 & 61.5 & 43.8 & 43.6 & 42.7 & 48.8 & 48.1 & 52.6 & 54.4 & 75.1 & 68.4 & 63.3 & 74.3 & 66.4 & 53.3 & 60.7 & 73.2 & 51.6 & 61.8 & 69.0 & 66.7 \\
\hline $\mathrm{Sm}$ & 6.8 & 6.8 & 7.3 & 7.6 & 10.6 & 7.9 & 8.0 & 7.8 & 8.5 & 9.2 & 8.8 & 10.8 & 14.5 & 13.2 & 10.5 & 12.4 & 11.6 & 9.6 & 11.0 & 13.1 & 10.2 & 10.9 & 11.5 & 11.7 \\
\hline $\mathrm{Eu}$ & 2.10 & 2.40 & 2.46 & 2.61 & 3.03 & 2.63 & 2.45 & 2.42 & 2.50 & 2.66 & 2.80 & 3.22 & 4.39 & 3.76 & 3.36 & 3.37 & 3.66 & 2.97 & 3.28 & 4.06 & 3.12 & 3.43 & 3.37 & 3.34 \\
\hline $\mathrm{Gd}$ & 5.51 & 6.11 & 6.16 & 7.01 & 8.0 & 7.28 & 6.92 & 6.71 & 7.32 & 7.59 & 7.83 & 8.73 & 12.74 & 9.38 & 9.40 & 8.99 & 9.38 & 7.55 & 8.70 & 9.64 & 8.29 & 8.95 & 9.38 & 8.10 \\
\hline $\mathrm{Tb}$ & 0.76 & 0.79 & 0.84 & 0.89 & 1.07 & 0.95 & 0.89 & 0.85 & 0.98 & 1.05 & 1.00 & 1.16 & 1.84 & 1.24 & 1.19 & 1.24 & 1.20 & 1.31 & 1.19 & 1.30 & 1.22 & 1.20 & 1.28 & 1.17 \\
\hline Dy & 3.97 & 4.28 & 4.48 & 4.83 & 5.54 & 4.59 & 4.56 & 4.07 & 4.91 & 5.72 & 4.83 & 6.15 & 9.10 & 6.78 & 5.99 & 6.46 & 6.37 & 5.46 & 6.39 & 7.13 & 6.46 & 6.40 & 6.25 & 6.43 \\
\hline Ho & 0.66 & 0.88 & 0.86 & 0.89 & 1.02 & 0.94 & 0.91 & 0.88 & 0.92 & 0.96 & 0.96 & 1.10 & 1.65 & 1.25 & 1.17 & 1.07 & 1.27 & 0.96 & 1.06 & 1.36 & 1.23 & 1.09 & 1.15 & 1.13 \\
\hline Er & 1.66 & 1.82 & 1.94 & 2.20 & 2.34 & 2.32 & 2.01 & 1.85 & 2.41 & 2.27 & 2.38 & 2.40 & 3.78 & 2.78 & 2.99 & 2.69 & 2.92 & 2.46 & 2.71 & 3.01 & 2.79 & 2.76 & 2.78 & 2.54 \\
\hline $\mathrm{Tm}$ & 0.23 & 0.21 & 0.24 & 0.28 & 0.32 & 0.27 & 0.28 & 0.23 & 0.30 & 0.32 & 0.31 & 0.27 & 0.49 & 0.35 & 0.37 & 0.38 & 0.40 & 0.34 & 0.37 & 0.37 & 0.40 & 0.39 & 0.38 & 0.36 \\
\hline $\mathrm{Yb}$ & 1.37 & 1.54 & 1.52 & 1.62 & 1.89 & 1.70 & 1.75 & 1.51 & 1.65 & 1.90 & 1.74 & 1.78 & 2.80 & 2.56 & 2.12 & 2.15 & 2.46 & 2.09 & 2.37 & 2.82 & 2.32 & 2.42 & 2.16 & 2.40 \\
\hline Lu & 0.19 & 0.23 & 0.21 & 0.25 & 0.25 & 0.24 & 0.26 & 0.24 & 0.24 & 0.28 & 0.28 & 0.24 & 0.40 & 0.35 & 0.30 & 0.25 & 0.36 & 0.30 & 0.33 & 0.39 & 0.24 & 0.34 & 0.34 & 0.34 \\
\hline
\end{tabular}

\begin{tabular}{|c|c|c|c|c|c|c|c|c|c|c|c|c|c|}
\hline $\begin{array}{l}\text { Lava type } \\
\text { Sample }\end{array}$ & $\begin{array}{l}\text { basalt } \\
\text { C5H }\end{array}$ & $\mathrm{C} 5 \mathrm{U}$ & $\mathrm{C} 10 \mathrm{R}$ & $00-5(2000)$ & $\mathrm{C} 5 \mathrm{~N}$ & $\mathrm{C} 9 \mathrm{P}$ & $00-6(2000)$ & 99-03 (1999) & $\mathrm{C} 8 \mathrm{~F}$ & $\mathrm{C} 8 \mathrm{~K}$ & C11D & C10Q & C10W(1982) \\
\hline $\mathrm{SiO}_{2}$ (\% wt.) & 43.37 & 46.16 & 45.79 & 44.69 & 46.20 & 47.11 & 45.55 & 45.44 & 44.88 & 46.66 & 44.23 & 44.76 & 44.33 \\
\hline $\mathrm{TiO}_{2}$ & 3.25 & 2.89 & 2.73 & 3.09 & 2.36 & 3.05 & 3.09 & 3.06 & 2.87 & 2.93 & 3.44 & 2.94 & 3.44 \\
\hline $\mathrm{Al}_{2} \mathrm{O}_{3}$ & 14.36 & 15.20 & 15.01 & 14.96 & 14.06 & 14.80 & 15.47 & 15.65 & 15.47 & 15.25 & 15.58 & 15.85 & 15.64 \\
\hline $\mathrm{Fe}_{2} \mathrm{O}_{3}$ & 13.24 & 12.22 & 12.04 & 12.53 & 12.08 & 11.65 & 12.41 & 12.58 & 11.90 & 11.98 & 13.39 & 12.04 & 13.47 \\
\hline $\mathrm{MnO}$ & 0.20 & 0.19 & 0.19 & 0.19 & 0.18 & 0.15 & 0.19 & 0.19 & 0.19 & 0.20 & 0.19 & 0.20 & 0.19 \\
\hline $\mathrm{MgO}$ & 7.48 & 7.04 & 7.17 & 7.04 & 6.99 & 6.81 & 6.72 & 6.49 & 6.29 & 6.28 & 5.98 & 5.93 & 5.90 \\
\hline $\mathrm{CaO}$ & 11.35 & 11.12 & 11.46 & 11.07 & 11.19 & 10.55 & 10.89 & 10.56 & 10.85 & 10.87 & 11.55 & 10.94 & 11.50 \\
\hline $\mathrm{Na}_{2} \mathrm{O}$ & 3.91 & 3.86 & 3.28 & 3.81 & 4.10 & 3.07 & 4.00 & 4.06 & 3.86 & 3.95 & 3.83 & 3.77 & 3.90 \\
\hline $\mathrm{K}_{2} \mathrm{O}$ & 1.58 & 1.26 & 1.30 & 1.46 & 1.32 & 1.30 & 1.59 & 1.59 & 1.76 & 1.45 & 1.37 & 1.57 & 1.37 \\
\hline $\mathrm{P}_{2} \mathrm{O}_{5}$ & 0.81 & 0.54 & 0.45 & 0.55 & 0.49 & 0.52 & 0.62 & 0.61 & 0.70 & 0.60 & 0.66 & 0.69 & 0.65 \\
\hline LOI & 0.03 & -0.50 & -0.26 & -0.47 & -0.13 & 0.71 & -0.55 & -0.19 & 0.73 & -0.36 & -0.61 & 0.90 & -0.70 \\
\hline Total & 99.58 & 99.98 & 99.16 & 98.92 & 98.84 & 99.72 & 99.98 & 100.04 & 99.50 & 99.81 & 99.61 & 99.59 & 99.69 \\
\hline $\mathrm{Be}(\mathrm{ppm})$ & 1.57 & 1.77 & 1.63 & 1.78 & 2.62 & 1.47 & 1.81 & 2.29 & 1.95 & 1.33 & 1.68 & 1.84 & 1.39 \\
\hline $\mathrm{Rb}$ & 41.8 & 35.8 & 30.6 & 35.4 & 46.2 & 33.0 & 35.9 & 37 & 46.8 & 31.3 & 35.3 & 35.6 & 33.0 \\
\hline $\mathrm{Sr}$ & 1143 & 1062 & 860 & 981 & 1174 & 776 & 998 & 1019 & 1055 & 820 & 1029 & 977 & 1004 \\
\hline Cs & 0.41 & 0.36 & 0.37 & 0.41 & 0.64 & 0.21 & 0.46 & 0.66 & 0.63 & 0.70 & 0.65 & 0.36 & 0.30 \\
\hline $\mathrm{Ba}$ & 492 & 450 & 372 & 433 & 604 & 383 & 453 & 475 & 556 & 381 & 450 & 480 & 441 \\
\hline V & 261 & 283 & 266 & 293 & 245 & 298 & 286 & 260 & 263 & 238 & 329 & 250 & 312 \\
\hline $\mathrm{Cr}$ & 160 & 206 & 262 & 218 & 153 & 272 & 181 & 141 & 189 & 203 & 73 & 126 & 83 \\
\hline $\mathrm{Co}$ & 37 & 41 & 43 & 44 & 39 & 37 & 41 & 39 & 37 & 33 & 41 & 33 & 39 \\
\hline $\mathrm{Ni}$ & 92 & 102 & 98 & 92 & 94 & 93 & 79 & 82 & 77 & 62 & 63 & 55 & 60 \\
\hline $\mathrm{Cu}$ & 58 & 96 & 75 & 82 & 88 & 37 & 74 & 81 & 81 & 52 & 107 & 70 & 105 \\
\hline $\mathrm{Zn}$ & 106 & 110 & 122 & 125 & 108 & 116 & 123 & 130 & 118 & 107 & 115 & 113 & 128 \\
\hline $\mathrm{Ga}$ & 23.0 & 24.5 & 21.7 & 23.6 & 23.3 & 24.7 & 23.8 & 23.90 & 23.0 & 21.5 & 25.0 & 22.0 & 24.7 \\
\hline $\mathrm{Ge}$ & 1.06 & 1.22 & 1.34 & 1.43 & 1.17 & 1.17 & 1.28 & 0.98 & 1.24 & 1.16 & 1.12 & 0.92 & 1.09 \\
\hline As & 1.14 & 1.14 & 1.08 & 1.17 & 1.74 & 0.52 & 1.18 & 1.03 & 1.34 & 0.91 & 1.16 & 1.23 & 1.57 \\
\hline $\mathrm{Cd}$ & $\mathrm{tr}$ & 0.14 & $\mathrm{tr}$ & $\mathrm{tr}$ & 0.14 & $\mathrm{tr}$ & $\operatorname{tr}$ & 0.50 & $\operatorname{tr}$ & 0.01 & 4.84 & $\mathrm{tr}$ & $\operatorname{tr}$ \\
\hline In & 0.06 & 0.06 & 0.11 & 0.10 & 0.07 & 0.15 & 0.10 & 0.10 & 0.13 & 0.07 & 0.08 & 0.13 & 0.06 \\
\hline Sn & 1.84 & 2.00 & 2.01 & 2.08 & 2.10 & 2.14 & 2.09 & 2.24 & 2.05 & 1.87 & 2.26 & 2.30 & 2.01 \\
\hline $\mathrm{Sb}$ & $\mathrm{tr}$ & 0.09 & $\mathrm{tr}$ & $\operatorname{tr}$ & 0.19 & 0.05 & $\mathrm{tr}$ & 0.13 & 0.15 & 0.03 & 0.11 & 0.16 & 0.01 \\
\hline Y & 29.6 & 31.1 & 25.9 & 29.2 & 32.4 & 30.2 & 30.3 & 31.4 & 28.5 & 27.0 & 31.2 & 27.8 & 30.4 \\
\hline $\mathrm{Zr}$ & 372 & 360 & 307 & 324 & 449 & 314 & 343 & 357 & 354 & 292 & 336 & 333 & 326 \\
\hline $\mathrm{Nb}$ & 100.0 & 88.4 & 74.2 & 82.6 & 116.2 & 58.5 & 86.9 & 87.9 & 100.2 & 73.3 & 82.5 & 93.2 & 78.9 \\
\hline Mo & 2.71 & 2.59 & 3.56 & 4.01 & 3.99 & 1.96 & 4.09 & 3.71 & 3.10 & 2.42 & 2.91 & 2.84 & 2.81 \\
\hline $\mathrm{Hf}$ & 8.47 & 8.05 & 6.66 & 7.27 & 9.83 & 7.48 & 7.41 & 7.45 & 7.72 & 6.39 & 7.29 & 7.30 & 7.74 \\
\hline
\end{tabular}




\begin{tabular}{|c|c|c|c|c|c|c|c|c|c|c|c|c|c|}
\hline $\mathrm{Ta}$ & 6.82 & 6.38 & 5.44 & 6.01 & 8.47 & 4.38 & 6.27 & 6.27 & 7.10 & 5.13 & 5.65 & 6.79 & 6.02 \\
\hline W & 0.63 & 0.94 & 1.00 & 1.19 & 1.27 & 0.57 & 1.22 & 1.25 & 1.10 & 0.85 & 1.06 & 0.99 & 1.08 \\
\hline $\mathrm{Pb}$ & 3.98 & 4.05 & 3.65 & 3.86 & 5.86 & 5.78 & 3.86 & 4.19 & 4.08 & 2.63 & 3.04 & 3.97 & 3.42 \\
\hline $\mathrm{Bi}$ & $\mathrm{tr}$ & $\mathrm{tr}$ & 0.06 & $\operatorname{tr}$ & $\operatorname{tr}$ & 0.00 & $\operatorname{tr}$ & 0.02 & 0.00 & $\mathrm{tr}$ & 0.04 & 0.05 & $\operatorname{tr}$ \\
\hline $\mathrm{Th}$ & 8.76 & 8.01 & 6.70 & 7.72 & 11.93 & 7.16 & 7.89 & 8.98 & 8.33 & 5.54 & 6.59 & 7.65 & 7.00 \\
\hline $\mathrm{U}$ & 2.16 & 2.13 & 1.85 & 1.97 & 3.36 & 1.32 & 2.04 & 2.02 & 2.15 & 1.43 & 1.70 & 2.00 & 1.85 \\
\hline $\mathrm{La}$ & 88.2 & 74.0 & 61.4 & 69.6 & 96.5 & 50.4 & 72.1 & 75.9 & 78.9 & 57.2 & 69.8 & 71.8 & 68.5 \\
\hline $\mathrm{Ce}$ & 168 & 146 & 123 & 139 & 183 & 106 & 143 & 153 & 156 & 119 & 143 & 140 & 142 \\
\hline Pr & 18.1 & 16.5 & 14.4 & 16.1 & 20.5 & 13.0 & 16.7 & 17.7 & 16.4 & 13.1 & 16.0 & 15.8 & 15.8 \\
\hline $\mathrm{Nd}$ & 74.3 & 66.4 & 53.3 & 60.7 & 73.2 & 51.6 & 61.8 & 69.0 & 66.7 & 52.7 & 62.4 & 58.5 & 62.1 \\
\hline $\mathrm{Sm}$ & 12.4 & 11.6 & 9.6 & 11.0 & 13.1 & 10.2 & 10.9 & 11.5 & 11.7 & 10.2 & 12.0 & 10.5 & 11.3 \\
\hline $\mathrm{Eu}$ & 3.37 & 3.66 & 2.97 & 3.28 & 4.06 & 3.12 & 3.43 & 3.37 & 3.34 & 2.96 & 3.42 & 3.24 & 3.69 \\
\hline $\mathrm{Gd}$ & 8.99 & 9.38 & 7.55 & 8.70 & 9.64 & 8.29 & 8.95 & 9.38 & 8.10 & 8.17 & 8.81 & 7.92 & 9.38 \\
\hline $\mathrm{Tb}$ & 1.24 & 1.20 & 1.31 & 1.19 & 1.30 & 1.22 & 1.20 & 1.28 & 1.17 & 1.07 & 1.22 & 1.04 & 1.28 \\
\hline Dy & 6.46 & 6.37 & 5.46 & 6.39 & 7.13 & 6.46 & 6.40 & 6.25 & 6.43 & 5.85 & 6.92 & 5.77 & 6.62 \\
\hline Ho & 1.07 & 1.27 & 0.96 & 1.06 & 1.36 & 1.23 & 1.09 & 1.15 & 1.13 & 1.04 & 1.17 & 1.08 & 1.28 \\
\hline Er & 2.69 & 2.92 & 2.46 & 2.71 & 3.01 & 2.79 & 2.76 & 2.78 & 2.54 & 2.39 & 2.74 & 2.40 & 2.91 \\
\hline $\mathrm{Tm}$ & 0.38 & 0.40 & 0.34 & 0.37 & 0.37 & 0.40 & 0.39 & 0.38 & 0.36 & 0.33 & 0.41 & 0.33 & 0.40 \\
\hline $\mathrm{Yb}$ & 2.15 & 2.46 & 2.09 & 2.37 & 2.82 & 2.32 & 2.42 & 2.16 & 2.40 & 2.09 & 2.29 & 2.35 & 2.51 \\
\hline $\mathrm{Lu}$ & 0.25 & 0.36 & 0.30 & 0.33 & 0.39 & 0.24 & 0.34 & 0.34 & 0.34 & 0.28 & 0.36 & 0.33 & 0.37 \\
\hline
\end{tabular}

\begin{tabular}{|c|c|c|c|c|c|c|c|c|c|c|c|c|c|c|c|c|c|c|}
\hline \multirow{2}{*}{$\begin{array}{l}\text { Rock type } \\
\text { Sample }\end{array}$} & \multicolumn{18}{|c|}{ Hawaiite } \\
\hline & $\mathrm{C} 9 \mathrm{R}$ & C1W(1959) & $\mathrm{C} 8 \mathrm{E}$ & C8C(1959) & $\mathrm{C} 10 \mathrm{~F}(1909)$ & $\mathrm{C} 10 \mathrm{~B}$ & 9903(1999) & 9901(1999) & $\mathrm{C9T}$ & C9Q & $\mathrm{C} 1 \mathrm{~B}(1$ & 1954) & $001(2000)$ & C9S & $\mathrm{C} 9 \mathrm{~V}$ & & C9Y & C9W2 \\
\hline $\mathrm{SiO}_{2}$ (\% wt.) & 46.45 & 45.62 & 48.16 & 45.41 & 46.39 & 46.29 & 47.13 & 47.3 & 47.36 & 47.96 & 47.45 & & 48.01 & 47.74 & 49.1 & & 49.34 & 49.79 \\
\hline $\mathrm{TiO}_{2}$ & 3.08 & 3.14 & 2.35 & 3.13 & 3.13 & 3.62 & 2.97 & 3 & 3.44 & 2.77 & 2.95 & & 2.94 & 3.55 & 2.65 & & 2.75 & 2.71 \\
\hline $\mathrm{Al}_{2} \mathrm{O}_{3}$ & 15.60 & 16.10 & 16.42 & 16.09 & 16.78 & 15.57 & 17.23 & 17.4 & 16.39 & 17.29 & 17.61 & & 17.64 & 17.00 & 17.2 & & 17.18 & 17.33 \\
\hline $\mathrm{Fe}_{2} \mathrm{O}_{3}$ * & 11.89 & 11.69 & 10.16 & 11.67 & 11.45 & 12.83 & 11.02 & 11.08 & 12.20 & 10.49 & 10.41 & & 10.33 & 11.70 & 10. & & 10.66 & 10.62 \\
\hline $\mathrm{MnO}$ & 0.18 & 0.19 & 0.17 & 0.20 & 0.19 & 0.18 & 0.2 & 0.2 & 0.19 & 0.19 & 0.20 & & 0.20 & 0.15 & 0.15 & & 0.14 & 0.15 \\
\hline $\mathrm{MgO}$ & 6.00 & 5.63 & 5.79 & 5.47 & 5.04 & 5.02 & 4.66 & 4.6 & 4.41 & 4.26 & 4.23 & & 4.21 & 4.14 & 4.0 & & 3.81 & 3.85 \\
\hline $\mathrm{CaO}$ & 10.36 & 10.33 & 9.62 & 10.22 & 9.81 & 10.26 & 9.43 & 9.48 & 9.69 & 8.89 & 8.88 & & 8.71 & 9.77 & 8.77 & & 8.85 & 8.94 \\
\hline $\mathrm{Na}_{2} \mathrm{O}$ & 3.94 & 4.64 & 4.30 & 4.56 & 4.83 & 3.64 & 4.95 & 4.99 & 4.08 & 4.36 & 5.35 & & 5.23 & 3.73 & 3.98 & & 4.14 & 4.17 \\
\hline $\mathrm{K}_{2} \mathrm{O}$ & 1.66 & 1.77 & 1.79 & 1.75 & 1.82 & 1.60 & 2.02 & 2.05 & 1.59 & 1.93 & 2.06 & & 2.16 & 1.55 & 1.7 & & 1.77 & 1.80 \\
\hline $\mathrm{P}_{2} \mathrm{O}_{5}$ & 0.68 & 0.81 & 0.63 & 0.80 & 0.85 & 0.66 & 0.76 & 0.74 & 0.64 & 0.78 & 0.88 & & 0.86 & 0.59 & 0.58 & & 0.58 & 0.58 \\
\hline L.O.I & -0.26 & -0.30 & 0.16 & -0.30 & -0.53 & 0.00 & -0.32 & -0.38 & -0.10 & 0.72 & -0.26 & & -0.28 & -0.16 & 0.9 & & -0.23 & -0.22 \\
\hline Total & 99.58 & 99.62 & 99.55 & 99.00 & 99.76 & 99.67 & 100.05 & 100.46 & 99.89 & 99.64 & 99.76 & & 100.01 & 99.76 & 99. & & 98.99 & 99.72 \\
\hline $\mathrm{Be}(\mathrm{ppm})$ & 1.55 & 1.70 & 2.22 & 1.87 & 2.09 & 1.42 & 2.17 & 1.83 & 1.68 & 1.81 & 1.95 & & 2.54 & 1.71 & 1.7 & & 1.74 & 1.93 \\
\hline $\mathrm{Rb}$ & 41.5 & 42.3 & 51.1 & 43.7 & 45.5 & 32.7 & 50 & 48 & 35.5 & 49.4 & 48.1 & & 48.5 & 37.1 & 53. & & 50.2 & 57.6 \\
\hline $\mathrm{Sr}$ & 991 & 1085 & 1007 & 1170 & 1095 & 976 & 1195 & 1137 & 890 & 1148 & 1155 & & 1181 & 980 & 837 & & 832 & 861 \\
\hline Cs & 0.41 & 0.41 & 0.79 & 0.40 & 0.37 & 0.34 & 0.97 & 0.74 & 0.43 & 0.43 & 0.52 & & 0.60 & 0.28 & 0.33 & & 0.34 & 0.62 \\
\hline $\mathrm{Ba}$ & 503 & 501 & 555 & 531 & 527 & 431 & 606 & 569 & 485 & 596 & 569 & & 591 & 465 & 463 & & 462 & 511 \\
\hline V & 262 & 257 & 200 & 258 & 240 & 307 & 231 & 206 & 279 & 214 & 204 & & 198 & 274 & 231 & & 227 & 234 \\
\hline $\mathrm{Cr}$ & 165 & 92 & 188 & 82 & 65 & 38 & 42 & 45 & 13 & 36 & 33 & & 22 & 28 & 42 & & 42 & 39 \\
\hline Co & 34 & 32 & 31 & 33 & 29 & 34 & 32 & 29 & 29 & 25 & 22 & & 24 & 28 & 25 & & 24 & 25 \\
\hline $\mathrm{Ni}$ & 77 & 54 & 81 & 53 & 38 & 25 & 35 & 27 & 16 & 29 & 18 & & 15 & 34 & 25 & & 24 & 26 \\
\hline $\mathrm{Cu}$ & 66 & 64 & 58 & 67 & 54 & 33 & 59 & 52 & 21 & 43 & 32 & & 30 & 23 & 28 & & 26 & 27 \\
\hline $\mathrm{Zn}$ & 111 & 117 & 103 & 111 & 120 & 124 & 137 & 128 & 122 & 116 & 109 & & 125 & 118 & 119 & & 103 & 118 \\
\hline $\mathrm{Ga}$ & 23.0 & 24.4 & 23.6 & 25.0 & 25.5 & 24.6 & 25.18 & 23.29 & 24.4 & 24.7 & 24.1 & & 24.9 & 36.2 & 26.2 & & 26.1 & 26.6 \\
\hline $\mathrm{Ge}$ & 1.06 & 1.20 & 1.29 & 1.12 & 1.19 & 1.11 & 1.03 & 0.86 & 1.10 & 1.12 & 1.11 & & 1.29 & 1.03 & 1.10 & & 1.05 & 1.15 \\
\hline As & 1.15 & 1.54 & 1.81 & 1.36 & 1.10 & 1.09 & 1.36 & 1.26 & 1.12 & 1.05 & 1.46 & & 1.40 & 1.15 & 1.70 & & 0.84 & 0.77 \\
\hline $\mathrm{Cd}$ & $\operatorname{tr}$ & 0.15 & 0.17 & 0.03 & $\operatorname{tr}$ & $\operatorname{tr}$ & 0.29 & 0.33 & $\operatorname{tr}$ & $\operatorname{tr}$ & 0.18 & & $\operatorname{tr}$ & 0.05 & $\operatorname{tr}$ & & $\operatorname{tr}$ & $\operatorname{tr}$ \\
\hline In & 0.15 & 0.06 & 0.05 & 0.08 & 0.17 & 0.14 & 0.10 & 0.12 & 0.14 & 0.06 & 0.05 & & 0.09 & 0.17 & 0.18 & & 0.07 & 0.05 \\
\hline $\mathrm{Sn}$ & 2.19 & 1.81 & 2.02 & 2.17 & 1.62 & 2.21 & 2.19 & 2.04 & 2.37 & 1.99 & 1.72 & & 2.32 & 2.37 & 2.40 & & 2.39 & 2.46 \\
\hline $\mathrm{Sb}$ & 0.16 & $\operatorname{tr}$ & 0.09 & 0.09 & 0.14 & 0.04 & 0.19 & 0.14 & 0.07 & 0.09 & $\operatorname{tr}$ & & $\operatorname{tr}$ & 0.08 & 0.15 & & 0.06 & 0.06 \\
\hline Y & 30.3 & 32.3 & 32.6 & 33.8 & 34.1 & 32.5 & 34.9 & 33.7 & 35.2 & 35.2 & 34.7 & & 35.5 & 31.5 & 34. & & 32.9 & 33.2 \\
\hline $\mathrm{Zr}$ & 362 & 369 & 409 & 385 & 397 & 367 & 366 & 329 & 355 & 383 & 407 & & 431 & 362 & 355 & & 357 & 374 \\
\hline $\mathrm{Nb}$ & 94.1 & 94.8 & 109.0 & 101.7 & 98.8 & 82.4 & 112.2 & 106.5 & 78.0 & 115.3 & 107.7 & & 121.5 & 76.9 & 72. & & 69.3 & 72.4 \\
\hline Мо & 3.08 & 3.54 & 4.21 & 3.55 & 3.90 & 2.94 & 4.61 & 4.16 & 2.63 & 2.70 & 3.98 & & 5.33 & 2.24 & 2.80 & & 2.27 & 2.14 \\
\hline $\mathrm{Hf}$ & 8.07 & 7.64 & 9.22 & 8.18 & 8.23 & 8.23 & 7.50 & 6.61 & 8.73 & 8.43 & 7.74 & & 8.70 & 8.36 & $8.0^{\top}$ & & 8.17 & 8.74 \\
\hline $\mathrm{Ta}$ & 7.02 & 6.77 & 7.97 & 7.37 & 7.09 & 6.06 & 7.55 & 7.64 & 6.05 & 8.38 & 7.51 & & 8.08 & 5.88 & 5.12 & & 4.95 & 5.28 \\
\hline W & 1.08 & 1.26 & 1.58 & 1.41 & 1.28 & 0.82 & 1.44 & 1.61 & 0.76 & 1.29 & 1.43 & & 1.58 & 0.83 & 0.70 & & 1.02 & 0.87 \\
\hline $\mathrm{Pb}$ & 4.13 & 3.76 & 4.80 & 4.43 & 1.80 & 4.93 & 4.88 & 4.86 & 9.43 & 4.85 & 3.55 & & 4.58 & 5.01 & 6.8 & & 6.19 & 6.54 \\
\hline $\mathrm{Bi}$ & 0.01 & $\operatorname{tr}$ & $\operatorname{tr}$ & $\operatorname{tr}$ & 0.01 & $\operatorname{tr}$ & 0.04 & 0.01 & $\operatorname{tr}$ & $\operatorname{tr}$ & $\operatorname{tr}$ & & $\operatorname{tr}$ & 0.01 & 0.0 & & $\operatorname{tr}$ & $\operatorname{tr}$ \\
\hline Th & 8.53 & 7.82 & 10.79 & 8.54 & 7.83 & 7.49 & 11.91 & 11.10 & 7.63 & 9.94 & 8.91 & & 10.09 & 7.52 & 8.77 & & 8.85 & 9.62 \\
\hline $\mathrm{U}$ & 2.27 & 2.15 & 2.70 & 2.35 & 2.19 & 1.89 & 2.64 & 2.46 & 2.01 & 2.43 & 2.50 & 2.75 & 1.85 & 2.1 & & 1.97 & 2.2 & \\
\hline $\mathrm{La}$ & 76.6 & 78.3 & 91.1 & 81.9 & 80.3 & 66.3 & 93.3 & 90.5 & 65.4 & 93.7 & 85.2 & 91.5 & 63.3 & 65. & & 65.4 & 68. & \\
\hline $\mathrm{Ce}$ & 154 & 155 & 167 & 168 & 169 & 138 & 183 & 178 & 132 & 190 & 169 & 182 & 134 & 140 & & 135 & 138 & \\
\hline $\operatorname{Pr}$ & 18.1 & 17.8 & 18.8 & 19.5 & 19.0 & 15.8 & 20.8 & 20.4 & 15.6 & 22.1 & 18.8 & 21.2 & 16.1 & 16. & & 15.3 & 15. & \\
\hline $\mathrm{Nd}$ & 66.0 & 71.1 & 68.8 & 75.3 & 73.1 & 67.6 & 80.1 & 79.0 & 60.1 & 79.2 & 74.1 & 77.7 & 61.1 & 65. & & 63.2 & 64. & \\
\hline $\mathrm{Sm}$ & 12.1 & 11.7 & 11.6 & 12.5 & 12.6 & 12.5 & 13.7 & 13.6 & 13.0 & 12.4 & 12.7 & 13.1 & 11.3 & 11. & & 11.6 & 12. & \\
\hline $\mathrm{Eu}$ & 3.69 & 3.48 & 3.29 & 3.96 & 3.81 & 3.38 & 3.84 & 3.83 & 3.82 & 3.76 & 3.84 & 4.02 & 3.65 & 3.3 & & 3.16 & 3.3 & \\
\hline Gd & 9.96 & 9.69 & 8.92 & 11.18 & 10.74 & 9.10 & 10.79 & 10.57 & 9.53 & 11.26 & 9.73 & 10.43 & 9.67 & 9.5 & & 8.55 & 8.9 & \\
\hline $\mathrm{Tb}$ & 1.29 & 1.28 & 1.21 & 1.34 & 1.45 & 1.28 & 1.38 & 1.40 & 1.36 & 1.45 & 1.34 & 1.41 & 1.26 & 1.2 & & 1.26 & 1.3 & \\
\hline Dy & 6.81 & 6.64 & 6.11 & 6.90 & 7.01 & 6.74 & 6.50 & 7.01 & 8.09 & 6.98 & 7.05 & 7.48 & 6.87 & 6.5 & & 6.34 & 7.0 & \\
\hline Ho & 1.31 & 1.26 & 1.23 & 1.38 & 1.34 & 1.18 & 1.32 & 1.30 & 1.46 & 1.42 & 1.30 & 1.28 & 1.35 & 1.3 & & 1.19 & 1.2 & \\
\hline $\mathrm{Er}$ & 2.91 & 3.12 & 3.01 & 3.35 & 3.09 & 3.08 & 3.06 & 3.10 & 3.40 & 3.61 & 3.10 & 3.32 & 3.25 & 3.4 & & 3.14 & 3.0 & \\
\hline $\mathrm{Tm}$ & 0.38 & 0.38 & 0.42 & 0.41 & 0.46 & 0.43 & 0.41 & 0.45 & 0.46 & 0.45 & 0.42 & 0.45 & 0.39 & 0.4 & & 0.41 & 0.4 & \\
\hline $\mathrm{Yb}$ & 2.41 & 2.44 & 2.63 & 2.45 & 2.45 & 2.57 & 2.52 & 2.46 & 3.15 & 2.44 & 2.60 & 2.83 & 2.42 & 2.6 & & 2.63 & 2.8 & \\
\hline $\mathrm{Lu}$ & 0.32 & 0.35 & 0.38 & 0.38 & 0.38 & 0.35 & 0.36 & 0.37 & 0.45 & 0.38 & 0.38 & 0.41 & 0.39 & 0.3 & & 0.38 & 0.3 & \\
\hline
\end{tabular}

The $\mathrm{K} / \mathrm{Ba}$ ratios (14-34) are low and show larger variations as the $\mathrm{K} / \mathrm{Rb}$ (355-495) ratios. Strontium varies from $1195 \mathrm{ppm}$ in the hawaiites to less than $600 \mathrm{ppm}$ in several Mg-rich basalt samples, with a majority of the values in the ranges 800 to $1000 \mathrm{ppm}$. Ba contents, low in basalts (259-392 ppm) are slightly higher in ha- waiites (441-606 ppm). Th/Ba, $\mathrm{Th} / \mathrm{Rb}$ and $\mathrm{Th} / \mathrm{U}$ ratios in $\mathrm{Mg}$-rich basalts are almost constant $(0.014-0.020,0.18-0.51$ and $3.55-3.72$ respectively). These ratios are rather similar and coincide with values reported for Pagalu in the oceanic section of the Cameroon Line (Lee et al. 1994), with typical HIMU oceanic 
island basalts (e.g., Tubuaii in the Pacific Ocean, Chauvel et al. 1992) and with average values reported for HIMU basalts: Th/Ba $=0.013-0.020, \mathrm{Th} / \mathrm{Rb}=0.20-0.29, \mathrm{Th} / \mathrm{U}=2.65-3.61$ (Weaver et al. 1991; Sun and McDonough, 1989).

The REE distributions of the basalts and hawaiites are quite similar with high $(\mathrm{La} / \mathrm{Yb})_{\mathrm{N}}$ ratios $(15-28)$ and $(\mathrm{La} / \mathrm{Sm})_{\mathrm{N}}$ ratios varying between 3.8 and 4.3. The patterns for the alkali lavas are in (Fig. 6) relatively steep and roughly parallel, showing relative LREE enrichment. The slightly positive Eu anomaly in hawaiites confirms an accumulation of plagioclase $\left(1.00<\mathrm{Eu} / \mathrm{Eu}^{*}<1.62\right)$. Samples C9U and C8C displays pattern with similar steep slope to those shown by other samples, but with two-times low contents (shifted of half towards the bottom). This particularity can be explained by the primitive character of the $\mathrm{C} 9 \mathrm{U}$ or by the incorporation of mantle material. Petrographic observations have shown that sample C9U includes numerous xenocrysts (olivine + Ca-rich pyroxene). Therefore, we suggest that the incorporation of these xenocrysts is responsible for the shift in pattern to low contents.

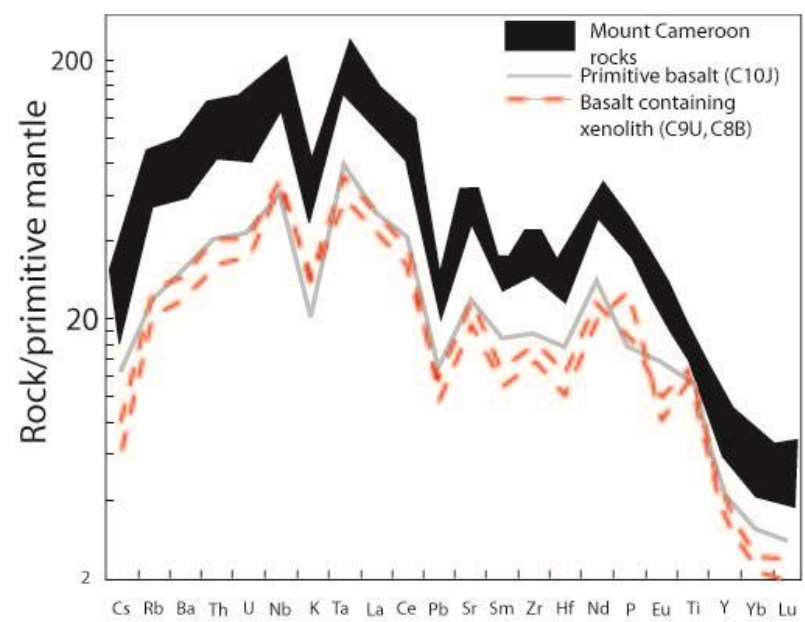

Fig. 6: Primitive Mantle-Normalized Multi-Element Diagrams for Rocks of Mt Cameroon.

Representative plots of the incompatible trace element concentrations for the Mt Cameroon lavas normalized to primitive mantle (McDonough and Sun, 1995) are presented in Fig. 7. All groups are characterized by a progressive enrichment from Lu to Ta and a relative depletion in the most incompatible elements (Cs to $\mathrm{U}$ ). These characteristics are similar to observations reported by Chauvel et al. (1992) in the case of HIMU-type alkali basalts, where enrichment is maximum for $\mathrm{Nb}-\mathrm{Ta}$ and the most incompatible trace elements $(\mathrm{Cs}, \mathrm{Rb}, \mathrm{Ba}, \mathrm{Th}, \mathrm{U}$ and $\mathrm{Nb})$ are less enriched. Differentiated samples (hawaiites) are characterized by the presence of pronounced positive $\mathrm{Zr}$ and negative $\mathrm{K}$ and $\mathrm{Pb}$ anomalies
A number of samples possess either small negative or small positive anomalies for $\mathrm{Zr}$ and $\mathrm{Ti}$ reflecting the fractionation (negative anomalies) or accumulation (positive anomalies) of minute aMts of Ti-rich phases. Ti and $\mathrm{Zr}$ negative anomaly can be explained also by amphibole-bearing mantle source (Spath et al. 2001).

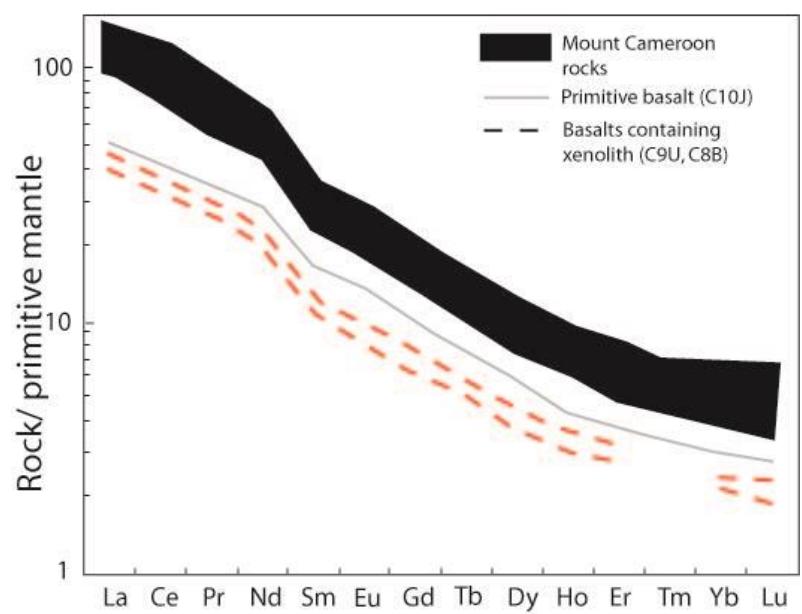

Fig. 7: Primitive Mantle-Normaliezed REE Digrams for Rocks of Mt Cameroon.

\section{2. $\mathrm{Sr}, \mathrm{Nd}$ and $\mathrm{Pb}$ isotopic ratios}

Preliminary isotope determinations of $\mathrm{Sr}-\mathrm{Nd}$ for alkali basalts and hawaiites fall in the ranges $0.70330-0.70335$ and 0.5128 0.5130 , respectively, plotting close to the reported values for the Cameroon Line alkali basalts (Halliday et al. 1988, 1990; Ngounouno et al. 2000, 2003) and for volcanoes from a number of oceanic islands, such as the St. Helena (Chaffey et al. 1989). In contrast, $\mathrm{Nd}$ isotopes determinations for the Mt Cameroon basalts show lower ${ }^{143} \mathrm{Nd} /{ }^{144} \mathrm{Nd}$ ratios than those of the basalts from other volcanoes of the Cameroon Line (Pagalu, Sao Tome, Principe, Manengouba) (Fig. 8).

$\mathrm{Pb}$ isotopes have higher unvarying values with 15.65 < ${ }^{207} \mathrm{~Pb} /{ }^{204} \mathrm{~Pb}<15.66$ overlapping the $\mathrm{Pb}$ isotopic data for the Cameroon Line. However, the Mt Cameroon basalts have highest ${ }^{206} \mathrm{~Pb} /{ }^{204} \mathrm{~Pb}$ and ${ }^{207} \mathrm{~Pb} /{ }^{204} \mathrm{~Pb}$ ratios $(20.2-20.5$ and $40.2-40.5)$ than those from other volcanic centers of the Cameroon Line (19-20 and 39-39.5 respectively) which suggest likely an implication of continental lithospheric mantle. The Mt Cameroon basalts with ${ }^{206} \mathrm{~Pb} /{ }^{204} \mathrm{~Pb}$ ratios > 20.2 lie close to the northern hemisphere reference line (NHRL) as defined by Hart (1984) which represents asthenospheric-derived MORB and OIB melts. 

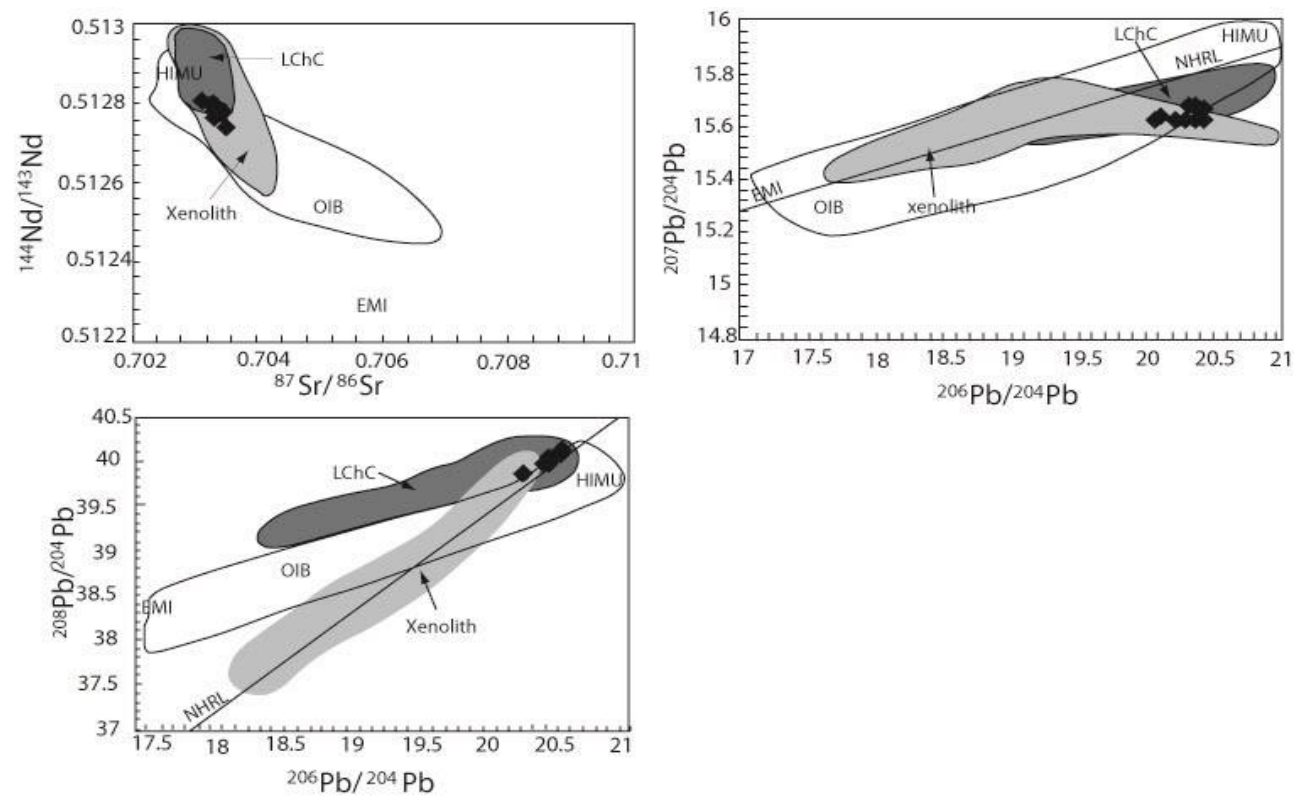

Fig. 8: $\left({ }^{87} \mathrm{Sr} /{ }^{86} \mathrm{Sr}\right) \mathrm{I}$ Vs. $\left({ }^{143} \mathrm{Nd} /{ }^{144} \mathrm{Nd}\right) \mathrm{I}(\mathrm{A}) ;\left({ }^{206} \mathrm{~Pb} /{ }^{204} \mathrm{~Pb}\right) \mathrm{I} \mathrm{Vs} .\left({ }^{87} \mathrm{Sr} /{ }^{86} \mathrm{Sr}\right) \mathrm{I}(\mathrm{B}) ;\left({ }^{206} \mathrm{~Pb} /{ }^{204} \mathrm{~Pb}\right) \mathrm{I}$ vs. $\left({ }^{207} \mathrm{~Pb} /{ }^{204} \mathrm{~Pb}\right) \mathrm{I}$ for Mt Volcanic Rocks (Data from This Study and from Yokoyama Et Al. (2007) . Literature Data for CHL where Compiled from Halliday Et Al. (1988, 1990), Lee Et Al. (1994), Marzoli Et Al. (1999, 2000), Yamgouot Et Al. (2005) And Nkouathio Et Al. (2008).Data for Xenolith from CHL are From Lee Etal. (1994).

\section{Discussion}

\subsection{Interpretation of mineral data}

Some important conclusions about the parental magma composition can be drawn from the presented data.

The forsterite content of olivine is related to the $\mathrm{MgO} / \mathrm{FeO}$ ratio of the parental magma; using a coefficient of 0.30 for the partition of $\mathrm{MgO} / \mathrm{FeO}$ between olivine and melt (Roeder and Emslie, 1970), the most primitive analysed olivine (Fo86) (similar to olivine in wherlite and clinopyroxenite xenoliths from the same volcano: Ngounouno et al. 2006) is in equilibrium with a melt with $\mathrm{MgO} / \mathrm{FeO}=2.5$. The composition of olivine primocrysts may, however, have been modified by equilibration with postcumulus overgrowth (Barnes, 1986; Cawthorn et al. 1992) as suggested by the more primitive compositions of olivine xenocrysts Fos8 in hawaiite $\mathrm{C} 1 \mathrm{~W}$. An estimation of the initial forsterite content can be made given the residual porosity in the Mg-rich basalts. If a maximum porosity of $35 \%$ in olivine phenocryst is assumed, an initial composition of Fo91 is suggested (Barnes, 1986), equivalent to an $\mathrm{MgO} / \mathrm{FeO}$ ratio of 2.5 in the liquid. This is in the range of primitive OIB (Wilson, 1989) and consistent with derivation from an enriched mantle source without significant differentiation. On this basis, the parental primary magma must have an $\mathrm{MgO}$ content of 13 wt. $\%$ to be able to crystallize an olivine of Fo91 composition. The composition of this estimated parental melt is appropriate to be in equilibrium with a lherzolite residue at about 1.6-1.7 $\mathrm{GPa}$ at a temperature of about $1400^{\circ} \mathrm{C}$ based on the experimental data of Falloon and Green (1988).

Ca-rich pyroxene from Mt Cameroon lavas is characterized by high $\mathrm{Ca}$ and low $\mathrm{Fe}^{2+}$, which correlate with both high $\mathrm{Al}^{\mathrm{IV}}$ and $\mathrm{Ti}$ compounds. This is consistent with (1) the alkaline nature of the magmas (low $\mathrm{SiO}_{2}$ ), (2) the near-liquidus crystallization of $\mathrm{Ca}$ rich pyroxene, (3) the relatively high $\mathrm{MgO}$ (> $5 \mathrm{wt} \%$ ) of magmas and $\mathrm{H}_{2} \mathrm{O}$, and $\mathrm{fO}_{2}$ (ca. NNO buffer).

Abundant Ca-rich amphibole microphenocrysts in Mg-rich basalts indicate that the Mt Cameroon magma had a relatively high $\mathrm{P}_{\mathrm{H} 2 \mathrm{O}}$, a feature that is also a characteristic of the Cameroon Line basaltic magmas (Déruelle et al. 1987; Nono et al. 1994; Ezangono et al 1995; Ngounouno et al. 2005). The melt from which these basalts formed was probably enriched in volatiles during their crystallization. Based on the considerations above, the parental magma could at the present stage be described as water-rich picritic basalt. This conclusion is based on experimental studies (Green, 1972) that have shown that in hydrous basaltic melts, amphibole can only exist as near-liquidus phases at pressures between 0.2 and $2 \mathrm{GPa}$ when $\mathrm{P}_{\mathrm{H} 2 \mathrm{O}}$ is greater than $0.5 \mathrm{P}_{\mathrm{T}}$.

The dominance of highly calcic plagioclase phenocrysts is strongly indicative of low-pressure igneous environments (Green and Ringwood, 1968; Yoder, 1969) (upper maximum of $0.5 \mathrm{GPa}$ ). Their crystallization in hawaiites indicates relatively high water content in the magma; in particular the order of appearance of Carich pyroxene-plagioclase implies that the system became progressively water-saturated (Nesbitt and Hamilton, 1970).

\subsection{Origin of xenocrysts and xenoliths hosted in basaltic lavas}

Some basalts of the Mt Cameroon contain xenocryts of Cr-spinel and Cr-diopside. Hawaiite C1W contains Fo8s olivine xenocrysts. The Cr-diopside xenocrysts of the Mt Cameroon basalts are similar in composition to those found in other alkali basalts (Ngounouno et al. 2000, 2003). Their restricted compositional range and the fact that their chemical composition is distinct from that of their host magma has led to the interpretation that these $\mathrm{Ca}$ rich pyroxenes are xenocrysts fragments derived from lithospheric peridotites. The Cr-diopside xenocrysts of Mt Cameroon are often fractured. They display sieved areas and have rounded shapes that could result from reaction with a hot magma after assimilation. Pressure and temperature estimates for crystallization of $\mathrm{Cr}$ diopside based upon phase relations in alkaline magmas (Bultitude and Green, 1971), the pMelts code (Ghiorso et al. 2002) and cpx-liquid thermobarometry (Putirka et al. 1996) are 1.7-2.3 GPa and $1200-1250^{\circ} \mathrm{C}$ respectively, corresponding to a depth of $35-40$ $\mathrm{km}$, that is to say within the spinel lherzolite field, just below the regional crust-mantle boundary $(28-30 \pm 6 \mathrm{~km}$ or $0.8-0.9 \pm 0.2$ GPa ; Poudjom Djomani et al. 1995).

As previously mentioned, ultramafic xenoliths are present in $\mathrm{Mt}$ Cameroon pyroclastic deposits. Studies on these xenoliths have shown that the upper mantle which underlies the Precambrian Mt Cameroon shield is heterogeneous and is composed of a variety of rock types, including wehrlite and clinopyroxenite (Ngounouno et al. 2006). Textural and mineralogical criteria from these studies have led to the conclusion that these rock types are crystalline cumulate resulting from crystallization of a basaltic magma (Ngounouno et al. 2006). This basaltic magma generated in the 
mantle may have incorporated accidental fragments of the spinelzone mantle during their rising through the subcontinental lithosphere. These rising magmas may have accumulated in reservoirs at the crust-mantle boundary $(28-30 \mathrm{~km})$. Here magmas may have undergone olivine, Ca-rich pyroxene, Fe-Ti oxides fractionation. Thus, the spinel-bearing xenoliths and xenocrysts, now preserved in Mt Cameroon flows as, may represent both the incorporated products of spinel-zone mantle fragmentation and the intratelluric products of spinel-fractionation.

\subsection{Nature of the source}

Some basalts from Mt Cameroon display stronger enrichments in $\mathrm{Nb}$ relative to LREE and LILE thus leading to low (LREE, LILE) $/ \mathrm{Nb}$ ratios $(\mathrm{La} / \mathrm{Nb}=0.78-0.87, \mathrm{Ba} / \mathrm{Nb}=5.25-6.50)$. The distinction between the different OIB end-members is better depicted in a $\mathrm{Ba} / \mathrm{Nb}-(\mathrm{Rb} / \mathrm{Nb}, \mathrm{K} / \mathrm{Nb})$ diagram, as these ratios are lower in HIMU than in EM sources and EMI is enriched in $\mathrm{Ba}$ with respect to EMII. The $\mathrm{Ce} / \mathrm{Pb}$ and $\mathrm{Nb} / \mathrm{U}$ ratios of the basalts from Mt Cameroon display similar ratios to those from Pagalu, Sao Tome and Principe islands $(\mathrm{Ce} / \mathrm{Pb}=35-40$ and $\mathrm{Nb} / \mathrm{U}=45$ 50). They have higher $\mathrm{Ce} / \mathrm{Pb}$ ratios $(37-48)$ than those obtained for MORB+OIB $(\approx 25 \pm 5$; Hofmann et al. 1986). The $\mathrm{Nb} / \mathrm{U}$ ratios (43-50) of the Mt Cameroon basalts approach the OIB+MORB value $(\approx 47 \pm 10)$, and are significantly higher than the values obtained for the continental crust $(\mathrm{Ce} / \mathrm{Pb} \approx 4$ and $\mathrm{Nb} / \mathrm{U} \approx 10)$. The Mt Cameroon basalts have $\mathrm{Nb} / \mathrm{La}$ ratios $(1.15-1.28)$ which place them near the HIMU values (Yokahoma et al. 2007).

The Mt Cameroon basalts have isotopic composition signatures which fall in the range of those described for the HIMU mantle (high ${ }^{206} \mathrm{~Pb} / 204 \mathrm{~Pb}$ ), ratio $(20.0-20.5)$, intermediate value of

$\left.{ }^{143} \mathrm{Nd} /{ }^{144} \mathrm{Nd}\right)$ i ratio $(0.5128)$, and low $\left({ }^{87} \mathrm{Sr} /{ }^{86} \mathrm{Sr}\right)$ i ratio $\left.(0.7031)\right)$. Many authors interpret these HIMU compositions as symptomatic of recycling of the subducted oceanic crust in the mantle (Weaver, 1991; Chauvel et al. 1992; Hofmann, 1997).

\subsection{Residual phases during partial melting}

The abundances of some diagnostics trace and major elements in basaltic liquids can be used to precise the mineralogical composition of their mantle source. $\mathrm{Mg}$ rich basalt from Mt Cameroon are depleted in $\mathrm{P}$ on normalized multi-element plots (Fig.7), possibly reflecting a small aMt of apatite in the source (Halliday et al. 1995). Zircon and sphene should be absent in the residue of the basalt suite as suggested by the incompatible behavior of elements such as Zr, light REE, and P (Fig. 6).

As previously stated, the depletion in $\mathrm{K}$ and $\mathrm{Rb}$ on the mantle normalized trace element patterns of basalts requires the presence of a potassic mineral in the residue of their mantle source, as noted by Ngounouno et al. (2005) for monchiquites from Tchircotché in the Upper Benue valley, northern Cameroon. Phlogopite has a mineral/melt partition coefficient for $\mathrm{Rb}$ and $\mathrm{K}$ higher than 1 in basaltic system (Foley et al. 1996). In the presence of fluorine, phlogopite is stable up to high pressures (6-7 GPa; Foley, 1986; Sudo and Tatsumi, 1990) where it decomposes to form numerous phases including potassic amphibole. The negative $\mathrm{K}$ and $\mathrm{Rb}$ anomalies in the normalized multi-element patterns of basalts suggest that the residual phase was phlogopite rather than amphibole. On the other hand, the absence of a marked negative anomaly in Ti could be the consequence of the presence of phlogopite in the source and the presence of a Ti-bearing phase such as kaersutite which has not remained in the residue.

As an alternative hypothesis, it has been suggested (i.e. Chauvel et al. 1992) that $\mathrm{K}$ negative anomaly is a characteristic of the source of the alkali basalts with HIMU Pb isotope signatures, such as the alkali basalts of the Cameroon Line (Halliday et al. 1990). Such relative $\mathrm{K}$ depletion could be related to the alteration and/or the deshydratation of the oceanic crust (in great depth $>110 \mathrm{~km}$ ) during an earlier subduction. In conclusion, the mantle source of the Mt Cameroon rocks probably contains amphibole, which is compatible with petrographic observations from lithospheric mantle xenolith studies from elsewhere in the Cameroon Line (Lee et al. 1996).

\subsection{Mantle metasomatism}

Petrographic data indicate that the studied ultramafic xenoliths (wehrlites and clinopyroxenites) from Mt Cameroon have equigranular and mosaic texture (Ngounouno et al. 2006). This suggests that "small-volume melt metasomatism" was ancient and followed by complete re-equilibration in the spinel-peridotite facies. This hypothesis is also supported by the petrological and geochgemical data of Lee et al. (1996), which evidenced that the spinel-mantle xenoliths from the continental Cameroon Line may represent fragments of subcontinental lithosphere formed at about the same time as the last major crust-forming event, (the PanAfrican) and were subsequently enriched by small melt fractions percolating through the upper mantle.

The ${ }^{87} \mathrm{Sr} /{ }^{86} \mathrm{Sr}$ ratios of ultramafic xenoliths from the Mt Cameroon Line are $\approx 0.70334$ whilst ${ }^{143} \mathrm{Nd} /{ }^{144} \mathrm{Nd}$ are $\approx 0.51272$ (Ngounouno et al. 2006), indicating that the underlying (Table 8) lithospheric mantle is heterogeneous and enriched. The overlap in $\mathrm{Sr}$ and $\mathrm{Nd}$ isotope values between the Mt Cameroon alkali basaltic magmas and their strongly metasomatised (amphibole veined) wehrlite and clinopyroxenite xenoliths overlap with their host basalts. Thus, it seems unlikely that the local infra-lithosphere has been a major component in the origin of the basalts and they are more probably formed from partial melting of deeper infra-lithospheric mantle.Metasomatized peridotites are considered to be representative of enriched subcontinental lithosphere. Melts forming K-rich amphibole are now considered to be a common metasomatic agent of the upper mantle, forming a network of thin dykes lacing mantle peridotite beneath zones of recent volcanism (e.g., Witt-Eickschen et al. 1998; Lee et al. 1996; Déruelle et al. 2001 ; Ngounouno et al. 2005). The common occurrence and the high modal proportions of amphibole in xenoliths from the upper mantle beneath the Mt Cameroon suggest that the lithospheric mantle has been metasomatized. We regard this as further evidence of a K-rich precursor metasomatic fluid in the infra-lithospheric mantle beneath the Mt Cameroon. The model of Green et al. (1994) predicts a region at a depth of 70-95 km in which metasomatism by reaction between "incipient melts" and garnet-spinel lherzolite will produce titaniferous pargasite in direct proportion to the aMt of introduced melt. The metasomatized lithosphere beneath the Mt Cameroon is enriched in clinopyroxene as illustrated by the formation of wehrlites and clinopyroxenites (olivine + clinopyroxene + chromite). The pyroxenites probably represent melts crystallized at mantle depth when the St. Helena mantle plume was active in this region.

\begin{tabular}{|c|c|c|c|c|c|c|c|c|c|c|c|c|}
\hline $\begin{array}{l}\text { Rock } \\
\text { type }\end{array}$ & Sample & ${ }^{87} \mathrm{Sr} /{ }^{86} \mathrm{Sr}$ & $\begin{array}{l}\mathrm{Rb} \\
(\mathrm{ppm})\end{array}$ & $\begin{array}{l}\mathrm{Sr} \\
(\mathrm{ppm})\end{array}$ & $2 \sigma$ & $\begin{array}{l}\mathrm{Nd} \\
(\mathrm{ppm})\end{array}$ & $\begin{array}{l}\mathrm{Sm} \\
(\mathrm{ppm})\end{array}$ & end & ${ }^{143} \mathrm{Nd} /{ }^{144} \mathrm{Nd}$ & ${ }^{206} \mathrm{~Pb} /{ }^{204} \mathrm{~Pb}$ & ${ }^{207} \mathrm{~Pb} /{ }^{204} \mathrm{~Pb}$ & ${ }^{208} \mathrm{~Pb} /{ }^{204} \mathrm{~Pb}$ \\
\hline \multirow{8}{*}{ Basalt } & $\mathrm{C} 10 \mathrm{~J}$ & 0.70333 & 14.3 & 473 & - & 35.1 & 6.8 & - & - & - & - & - \\
\hline & $\mathrm{C} 9 \mathrm{U}$ & 0.70342 & 12.6 & 382 & 0.00002 & 24.9 & 4.4 & 1.99 & 0.51274 & 20.267 & 15.66 & 40.15 \\
\hline & C8B & 0.70338 & 15.6 & 477 & 0.00001 & 28.6 & 5.08 & 2.01 & 0.51274 & 20.264 & 15.65 & 40.14 \\
\hline & C10R & 0.70342 & 12.21 & 394 & - & 24.7 & 4.7 & 1.99 & 0.51274 & 20.27 & 15.66 & 40.15 \\
\hline & $\mathrm{C} 10 \mathrm{~F}$ & 0.70331 & 35.90 & 1065 & - & 73.6 & 12.9 & 3.24 & 0.51280 & 20.394 & 15.65 & 40.12 \\
\hline & $\mathrm{C} 8 \mathrm{~N}$ & 0.70335 & 32.9 & 1004 & - & 69.1 & 11.8 & 2.69 & 0.51278 & 20.281 & 15.64 & 40.03 \\
\hline & $\mathrm{C} 8 \mathrm{C}$ & 0.70332 & 33.6 & 1071 & - & 74.7 & 12.8 & 2.97 & 0.51279 & 20.313 & 15.64 & 40.04 \\
\hline & C10W & 0.70332 & 32.9 & 995 & - & 62.8 & 11.2 & 2.85 & 0.51278 & 20.342 & 15.64 & 40.10 \\
\hline
\end{tabular}




\subsection{Geodynamic implications}

The above melting model for the Mt Cameroon rocks as well as their HSFE, LREE, LILE ratios and Sr-Nd isotopic signature allow the identification of a HIMU-like component in the generation of the basaltic series. If the participation of the above mentioned mantle source component in the petrogenesis of the Mt Cameroon lavas is accepted, the magmatism in this region could develop in two steps. In a first stage, an asthenospheric diapir with trace element and isotopic ratios similar to the HIMU-, OIB-reservoir would trigger magma generation in the overlying subcontinental lithosphere by melting of pervasive enriched streaks or blobs with amphibole, giving rise to primitive liquids. The presence of residual amphibole requires melting close to the asthenosphere lithosphere boundary or within the lithospheric mantle. In subsequent steps the mantle diapir head would start to melt, the lithosphere becoming stripped of this enriched component, and produced basaltic liquids with $\mathrm{Sr}-\mathrm{Nd}$ isotopic ratios closer to the OIB component. These data cannot be interpreted in terms of a mantle plume, because the diameter of the Mt Cameroon $(<100 \mathrm{~km})$ and the narrow zone of lithospheric thinning $(<100 \mathrm{~km})$ preclude mantle upwelling from any great depth. It is therefore likely that this is simply a localized diapiric instability within the upper asthenospheric and the HIMU-like Sr-Nd isotopic characteristics are inherited from a zone at the base of the continental lithosphere. The ascent of an asthenospheric diapir and the triggering of magma generation also involve some additional geodynamic implications. The melting of the head of the diapir can be produced if it decompresses likely in Mt Cameroon, indicate a slight crustal thinning (28-30 km) and the sedimentary evolution reflects an extensional regime

\section{Conclusions}

Voluminous mafic magmatism occurred in the Mt Cameroon at the ocean-continent boundary of the Cameroon Line. This resulted in the formation of a restricted variety of lava types (basalts and hawaiites) that were extruded during late Miocene to Present time. Intermediate and evolved compositions are absent. The Mt Cameroon also contains wehrlite and clinopyroxenite xenoliths. The primitive parental magma results from primary magmas of HIMUlike character, generated by small degrees of partial melting of an infra-lithospheric metasomatized. This mantle source has geochemical characteristics comparable to the source which generated alkaline basaltic magmas all along the Cameroon Line. The geochemical diversity of mantle xenoliths observed in alkaline volcanic rocks from the Mt Cameroon suggests that metasomatic enrichment processes during the Mesozoic produced substantial chemical heterogeneity in the lithospheric mantle beneath $\mathrm{Mt}$ Cameroon. These inferred processes led to an enrichment of incompatible elements by percolating hydrous fluids, which resulted in the formation of hydrous mineral phases in the lithospheric mantle.

\section{Acknowledgments}

The Professor Albert Jambon is acknowledged for providing a grant to Y.F for a one-month stay in France in the 'Laboratoire de Magmatologie et de Géochimie Inorganique et Expérimentale (MAGIE), 'Université Pierre-et-Marie-Curie', Paris 6. The isotopic measurements at the Université Libre de Bruxelles were financially supported by the Ministère des Affaires Economiques (Project SGB/NAT 91-98).

\section{References}

[1] Ashwal LD, Demaiffe D, Torsvik TH (2002) Petrogenesis of Neoproterozoic granitoids and related rocks from the Seychelles: the case for an Andean-type arc origin. J. Petrol. 43, 45-73. https://doi.org/10.1093/petrology/43.1.45.

[2] Barnes SJ (1986). The effect of trapped liquid crystallization on cumulus mineral compositions in layered intrusions. Contrib. Mineral. Petrol. 93,524-531 https://doi.org/10.1007/BF00371722.

[3] Bultitude RJ, Green DH (1971) Experimental study of crystalliquid relationships at high pressures in olivine nephelinite and basanite compositions. J Petrol 12,121-147 https://doi.org/10.1093/petrology/12.1.121.

[4] Bonatti E, Harrisson CGA (1976) Hot lines in the Earth's mantle. Nature 263, 402-404. https://doi.org/10.1038/263402a0.

[5] Carignan J, Hild P, Mevelle G, Morel J, Yeghicheyan D (2001) Routine analyses, of trace elements in geological samples using flow injection and low pressure on line liquid chromatography coupled to ICP-MS: a study of geochemical reference materials BR, DR-N, UB-N, AN-G and GH. Geostandards Newsletters 25, $187-$ 198. https://doi.org/10.1111/j.1751-908X.2001.tb00595.x.

[6] Chauvel C, Hofmann AW, Vidal P (1992) HIMU-EM: the French Polynesian connection, Earth Planet. Sci. Lett. 110, 99-119. https://doi.org/10.1016/0012-821X(92)90042-T.

[7] Cawthorn RG, Sander BK, Jones IM (1992) Evidence for the trapped liquid shift effect in the Mt Ayliff Intrusion, South Africa. Contrib. Mineral. Petrol. 111,194-202 https://doi.org/10.1007/BF00348951.

[8] Déruelle B, N'ni J, Kambou R (1987) Mt Cameroon: an active volcano of the Cameroon Line. J. Afr. Earth Sci. 6, 197-214.

[9] Déruelle B, Moreau C, Nkoumbou C, Kambou R, Lissom E, Njongfang J, Ghogomu RT, Nono A (1991) The Cameroon Line: a review, in: A.B. Kampunzu, R.T. Lubala (Eds.), Magmatism in Extensional Structural Settings. The Phanerozoic African Plate, Springer-Verlag, Berlin, pp. 274-327. https://doi.org/10.1007/9783-642-73966-8 12.

[10] Droop GTR (1987) A general equation for estimating $\mathrm{Fe}^{3+}$ concentrations in ferromagnesian silicates and oxides from microprobe analyses, using stoichiometric criteria. Mineral. Mag. 51,431-5. https://doi.org/10.1180/minmag.1987.051.361.10.

[11] Ézangono J, Déruelle B, Ménard JJ, (1995) Benmoreites from Tchabal Djinga volcano (Adamawa, Cameroon), products of kaersutite + plagioclase assimilation by a trachytic magma. Terra. Abst. Suppl. Terra Nova 7: 161.

[12] Falloon TJ, Green DH, Hatton CJ, Harris KL (1988) Anhydrous partial melting of fertile and depleted peridotite from 2 to $30 \mathrm{kbar}$ and application to basalt petrogenesis: J. Petrol. 29, 257-282. https://doi.org/10.1093/petrology/29.6.1257.

[13] Foley SF, Taylor WR, Green DH (1986) the role of fluorine and oxygen fugacity in the genesis of the ultra-potassic rocks. Contrib. Mineral. Petrol. 94, 183-192. https://doi.org/10.1007/BF00592935.

[14] Foley SF, Jackson SE, Fryer BJ, Greenough JD, Jenner GA (1996) Trace element partition coefficients for clinopyroxene and phlogopite in an alkaline lamprophyre from New found land by LAMICP-MS. Geochim. Cosmochim. Acta 60 629-63. https://doi.org/10.1016/0016-7037(95)00422-X.

[15] Ghiorso MS, Hirschmann MM, Reiners PW, Kress VC (2002) The pMELTS: A revision of MELTS for improved calculation of phase relations and major element partitioning related to partial melting of the mantle to $3 \mathrm{GPa}$. Geochem Geophy Geosy 3:1030 https://doi.org/10.1029/2001GC000217.

[16] Green TH (1972) Crystallization of calcalkaline andesite under controlled high-pressure hydrous conditions. Contrib. Mineral. Petrol. 34, 150-166. https://doi.org/10.1007/BF00373770.

[17] Green TH (1994) Experimental studies of trace-element partitioning applicable to igneous petrogenesis - Sedona 16 years later. Chem. Geol. 117, 1-36. https://doi.org/10.1016/00092541(94)90119-8.

[18] Green DH, Ringwood AE (1967) TIle genesis of basaltic magmas. Petrol. 15 , $103-190$ https://doi.org/10.1007/BF00372052.

[19] Halliday AN, Dickin AP, Fallick AE, Fitton JG (1988) Mantle dynamics: a $\mathrm{Nd}, \mathrm{Sr}, \mathrm{Pb}$ and $\mathrm{O}$ isotopic study of the Cameroon Line volcanic chain. J. Petrol. 29, 181-211. Halliday, A.N., Davidson, J.P., Holden, P., DeWolf, C., Lee, D.C., Fitton, J.G., 1990. Trace 
fractionation in plumes and the origin of HIMU mantle beneath the Cameroon line. Nature 347, 523-528. https://doi.org/10.1038/347523a0.

[20] Hofmann, A.W., Jochum, K.P., Seufert M., White, W.M., 1986. Nb and $\mathrm{Pb}$ in oceanic basalts: new constraints on mantle evolution. Earth Planet. Sci. Lett. 79, 33-45. https://doi.org/10.1016/0012821X(86)90038-5.

[21] Leake, B.E., and 20 co-authors. 1997. Nomenclature of amphiboles: report of the subcommitee on amphiboles of the International Mineralogical Association commission on new minerals and mineral names. Mineral. Mag. 61, 295-321 https://doi.org/10.1180/minmag.1997.061.405.13.

[22] Lee, D.C., Halliday, A.N., Fitton, J.G., Poli, G., 1994. Isotopic variations with distance and time in the volcanic islands of the Cameroon Line - evidence for a mantle plume origin. Earth Planet. Sci Lett. 123, 119-138. https://doi.org/10.1016/0012-821X(94)902623 .

[23] Marzoli, A., Renne, P.R., Piccirillo, E.M., Francesca, C., Bellien, G., Mel, A.J., Nyobe, J.B., N'ni, J., 1999. Silicic magmas from the continental Cameroon Volcanic Line (Oku, Bambouto and Ngaoundéré): ${ }^{40} \mathrm{Ar} /{ }^{\beta 9} \mathrm{Ar}$ dates, petrology, $\mathrm{Sr}-\mathrm{Nd}-\mathrm{O}$ isotopes and their petrogenetic significance. Contrib. Mineral. Petrol. 135,133-150. https://doi.org/10.1007/s004100050502.

[24] Marzoli, A., Piccirillo, E.M., Renne, P.R., Bellieni, G., Lacumin, M., Nyobe, J.B., Tngwa, A.T., 2000. The Cameroon Volvanic Line revisited: petrogenesis of continental basaltic magmas from lithospheric and asthenospheric mantle sources. J. Petrol. 41, 87-109. https://doi.org/10.1093/petrology/41.1.87.

[25] Maury, R. C., Defant, M. J., Joron, J. L., 1992. Metasomatism of the sub-arc mantle inferred from trace elements in Philippine xenoliths. Nature 360, p. 661-663. https://doi.org/10.1038/360661a0.

[26] McDonough, W., Sun, S.S., 1995. The composition of the Earth, Chem. Geol. 120, 223-253. https://doi.org/10.1016/00092541(94)00140-4.

[27] Middlemost, E.A.K., 1989. Iron oxidation ratios, norms and the classification of volcanic rocks. Chem. Geol. 77, 19-26 https://doi.org/10.1016/0009-2541(89)90011-9.

[28] Moreau, C., Regnoult, J.M., Déruelle, B., Robineau, B., 1987. A new tectonic model for the Cameroon Line, Central Africa, Tectonophysics 139-141 317-334. https://doi.org/10.1016/00401951(87)90206-X.

[29] Morimoto, N., 1989. Nomenclature of pyroxenes. Can. Mineral. 27, 143-156. https://doi.org/10.2465/minerj.14.198.

[30] Nesbitt, R.W., Hamilton, D.L., 1970. Crystallization of an alkali olivine basalt under controlled $\mathrm{PO}_{2}-\mathrm{PH}_{2} \mathrm{O}$, conditions. Phys. Earth Planet. Inter. 3, 309-315. https://doi.org/10.1016/00319201(70)90067-1.

[31] Ngounouno, I., Déruelle, B., Demaiffe, D., 2000. Petrology of the bimodal Cenozoic volcanism of the Kapsiki plateau (northern most Cameroun, central Africa), J. Volcanol. Geotherm. Res. 102, 21-44 https://doi.org/10.1016/S0377-0273(00)00180-3.

[32] Ngounouno, I., Déruelle, B., Demaiffe, D., Montigny, R., 2003. Petrology of the Cenozoic volcanism in the Upper Benue valley, northern Cameroon (Central Africa), Contrib. Mineral. Petrol. 145 , 87-106. https://doi.org/10.1007/s00410-002-0438-6.

[33] Ngounouno, I., Déruelle, B., Montigny, R., Demaiffe, D., 2005. Petrology and geochemistry of monchiquites from Tchircotché (Garoua rift, north Cameroon, central Africa), Mineral. Petrol. 83, 167190. https://doi.org/10.1007/s00710-004-0068-y.

[34] Ngounouno, I., Déruelle, B., Montigny, R., Demaiffe, D., 2006. Les camptonites du mont Cameroun, Cameroun, Afrique, C. R. Geoscience 338, 537-544. https://doi.org/10.1016/j.crte.2006.03.015.

[35] Nimis P., Ulmer P. (1998). Clinopyroxene geobarometry of magmatic rocks. Part 1: An structural geobarometer for anhydrous and hydrous, basic and ultrabasic systems. Contrib. Mineral. Petrol. 133,122-135. https://doi.org/10.1007/s004100050442.

[36] Nkouathio, D.G., Ménard, J.-J., Wandji, P., Bardintzeff, J.-M., 2002. The Tombel graben (West Cameroon): a recent monogenetic volcanic field of the Cameroon Line. J. Afr. Earth Sci. 35: 285-300. https://doi.org/10.1016/S0899-5362(02)00031-3.

[37] Nkoumbou, C., Déruelle, B., Velde, D., 1995. Petrology of Mt Etinde nephelinite series, J. Petrol. 36, 373. https://doi.org/10.1093/petrology/36.2.373.

[38] Nono, A., Déruelle, B., Demaiffe, D., Kambou, R., 1994. Tchaba Nganha volcano in Adamawa (Cameroon): petrology of a continental alkaline lava series. J. Volcanol. Geotherm. Res. 60, 147-178. https://doi.org/10.1016/0377-0273(94)90066-3.

[39] Pouchou, J.L., Pichoir, F., 1991. Quantitative analysis of homogeneous or stratified microvolumes applying the model "PAP". In: Heinrich KFJ, Newbury DE (eds) Electron probe quantification.
Plenum Press, New York, 31-75. https://doi.org/10.1007/978-1 4899-2617-3_4.

[40] Poudjom, Djomani, Y.H., J.M. Nnange, M., Diament, G.J., Ebinger, J.D., Fairhead, 1995. Effective elastic thickness and crustal thickness variations in West-Central Africa inferred from gravity data. J.
Geophys.
Res.
100
$22047-22070$ https://doi.org/10.1029/95JB01149.

[41] Puturirka, K., Johnson, M., Kinzler, R., Longhi, J., Walker, D., 1996. Thermobarometry of mafic igneous rocks based on clinopy roxene-liquid equilibria, $0 \pm 30 \mathrm{kbar}$. Contrib. Mineral. Petrol. 123, 92-108 https://doi.org/10.1007/s004100050145.

[42] Presnall, D.C., Dixon, S.A., Dixon, J.R., O'Donnell, T.H., Brenner, N.L., Schrock, R.L., Dycus, D.W. 1978. Liquidus phase relations on the join diopside-forsterite-anorthite from $1 \mathrm{~atm}$ to $20 \mathrm{kbar}$ : their bearing on the generation and crystallization of basaltic magma Contrib. Mineral. Petrol. 66,203-220 https://doi.org/10.1007/BF00372159.

[43] Thornton, C.P., Tuttle, O.F., 1960. Chemistry of igneous rocks-[Part] 1 I. Differentiation index. Amer. J. Sci. 258: 664-684. https://doi.org/10.2475/ajs.258.9.664.

[44] Roeder, P.L., Emslie, R.F., 1970. Olivine-liquid equilibrium. Con$\begin{array}{lll}\text { trib. } & \text { Mineral. } & \text { 29, }\end{array}$ https://doi.org/10.1007/BF00371276.

[45] Simkin, T., Smith, J.V., 1970. Minor-element distribution in olivine. J. Geol. 78, 304-325. https://doi.org/10.1086/627519.

[46] Späth, A., Le Roex, A.P., Opiyo-Akech, N., 2001. Plume - Lithopheric interaction and origin of continental rift-related alkaline volcanism- The Chyulu Hills Volcanic Province, Southern Kenya. J. Petrol. 42, 765-787. https://doi.org/10.1093/petrology/42.4.765.

[47] Spencer, K.J., Lindsley, D.H., 1981. A solution model for coexisting ion-titanium oxides. Amer. Mineral. 66, 1189-1201.

[48] Stormer, C.F., 1983.The effects of recalculationon estimates of temperature and oxygen fugacity from analyses of multicomponent iron-titanium oxides. Am. Mineral. 68, 586-594.

[49] Sudo, A, Tatsumi Y. (1990). Phlogopite and K-amphibole in the upper mantle: Implication for magma genesis in subduction $\begin{array}{lllll}\text { zones.Geophs. } & \text { Res. } & \text { Lett. } & 17, & 29-32 .\end{array}$ https://doi.org/10.1029/GL017i001p00029.

[50] Sun, S.S., McDonough, W.F., 1989. Chemical and isotopic systematics of oceanic basalts: implications for mantle composition and processes. In: Saunders AD and Norry MJ (eds) Magmatism in the oceans basins. Geol Soc London Spec. Publ. 42, 313-345. https://doi.org/10.1144/GSL.SP.1989.042.01.19.

[51] Temdjim, R., 2005. Contribution à la connaissance du manteau supérieur du Cameroun au travers de l'étude des enclaves ultrabasiques et basiques remontées par les volcans de Youkou (Adamaoua) et de Nyos (Ligne du Cameroun). Thèse Doct. État. Univ. Yaoundé 1, $339 \mathrm{pp}$.

[52] Wass, S.Y. 1979. Multiple origins of clinopyroxene in alkali basaltic rocks, Lithos 12, 115-132. https://doi.org/10.1016/0024 4937(79)90043-4.

[53] Weaver, B.L. 1991. Trace element evidence for the origin of oceanic island basalts. Geology 19, 123-126. https://doi.org/10.1130/00917613(1991)019<0123:TEEFTO >2.3.CO;2.

[54] Wilson, M., 1989. Igneous petrogenesis. Chapman and Hall, London, 466 p. https://doi.org/10.1007/978-1-4020-6788-4.

[55] Witt-Eickschen, G., W. Kaminskry, U, Kramm, B., Harte, 1998. The nature of young vein metasomatism in the lithosphere of the West Eifel (Germany): geochemical and isotopic constraints from composite mantle xenoliths from the Meerfelder maar. J. Petrol. 39, 155-185. https://doi.org/10.1093/petroj/39.1.155.

[56] Yoder, H.S J., Tilley, C.E., 1962. Origin of basalt magmas: an experimental study of natural and synthetic rock systems. J. Petrol. 3 , 342-532. https://doi.org/10.1093/petrology/3.3.342.

[57] Yokahoma, T., Aka, T.F., Kusakabe M., Nakamura, E., 2007. Plume-lithosphere interaction beneath Mt. Cameroon volcano, West Africa: Contraints from 238U-230Th-236Ra and $\mathrm{Sr}-\mathrm{Nd}-\mathrm{Pb}$ isotope systematic, Geochim. Cosmochim.acta, doi:10.1016/j.gca.01.010. 